\title{
Some experimental constraints for spectral parameters used in the Warner and McIntyre gravity wave parameterization scheme
}

\author{
M. Ern ${ }^{1}$, P. Preusse ${ }^{1}$, and C. D. Warner ${ }^{2}$ \\ ${ }^{1}$ Institute for Stratospheric Research (ICG-I), Forschungszentrum Juelich, Juelich, Germany \\ ${ }^{2}$ Centre for Atmospheric Science, University of Cambridge, Cambridge, UK
}

Received: 8 March 2006 - Published in Atmos. Chem. Phys. Discuss.: 14 June 2006

Revised: 1 September 2006 - Accepted: 17 September 2006 - Published: 27 September 2006

\begin{abstract}
In order to incorporate the effect of gravity waves (GWs) on the atmospheric circulation most global circulation models (GCMs) employ gravity wave parameterization schemes. To date, GW parameterization schemes in GCMs are used without experimental validation of the set of global parameters assumed for the GW launch spectrum. This paper focuses on the Warner and McIntyre GW parameterization scheme. Ranges of parameters compatible with absolute values of gravity wave momentum flux (GW-MF) derived from CRISTA- 1 and CRISTA-2 satellite measurements are deduced for several of the parameters and the limitations of both model and measurements are discussed. The findings presented in this paper show that the initial guess of spectral parameters provided by Warner and McIntyre (2001) are some kind of compromise with respect to agreement of absolute values and agreement of the horizontal structures found in both measurements and model results. Better agreement can be achieved by using a vertical wavenumber launch spectrum with a wider saturated spectral range and reduced spectral power in the unsaturated part. However, even with this optimized set of global launch parameters not all features of the measurements are matched. This indicates that for further improvement spatial and seasonal variations of the launch parameters should be included in GW parameterization schemes.
\end{abstract}

\section{Introduction}

Gravity waves (GWs) are one of the most important vertical coupling processes in the atmosphere transferring momentum from the troposphere into the stratosphere and mesosphere and contributing to the acceleration and deceleration of the horizontal wind. A review of GW dynamics

Correspondence to: $\mathrm{M}$. Ern

(m.ern@fz-juelich.de) has been given by Fritts and Alexander (2003). Since the spatial resolution in global circulation models (GCMs) is not sufficient to resolve medium scale processes like gravity waves the contribution of GWs to the model dynamics is calculated with GW parameterization schemes. Different approaches have led to a number of different parameterization schemes (e.g., Lindzen, 1981; McFarlane, 1987; Medvedev and Klaassen, 1995; Hines, 1997a,b; Warner and McIntyre, 2001) with spectral parameterizations being the latest development. A review about GW parameterization schemes is given by Kim et al. (2003).

Parameterizing instead of resolving GW processes in a GCM allows the use of model grids with lower spatial resolution and reduces the computational cost dramatically, a requirement which is important especially for long-term model runs. In spectral GW parameterization schemes a GW spectral distribution (launch spectrum) is launched at a fixed altitude (launch altitude). Then the wave spectrum is propagated vertically through the background atmosphere. There are several parameters that are more or less freely adjustable. One parameter is the launch altitude itself. Other parameters define the spectral shape of the GW spectrum at the launch altitude.

There are some assumptions about the properties of the GW spectrum which are commonly made. Supported by various observations (e.g., Sato, 1994; Nastrom et al., 1997; Cot, 2001; Hertzog and Vial, 2001; Hertzog et al., 2001, 2002; Tsuda et al., 2004) the intrinsic frequency $\hat{\omega}$ spectrum of the GW total wave energy density often is assumed to decrease with $\hat{\omega}^{-p}$ for large values of $\hat{\omega}$ and typically a value of $p=5 / 3$ is used.

Also well-constrained is the vertical wavenumber $m$ spectrum in its saturated part at large vertical wavenumbers $m$ : there are numerous observations that the saturated part of the vertical wavenumber spectrum obeys a power law $\sim m^{-t}$ and decreases with a power of $t \approx 3$ (e.g., VanZandt, 1982; Tsuda et al., 1989, 1991; Sato, 1993, 1994; Allen and Vin-

Published by Copernicus GmbH on behalf of the European Geosciences Union. 


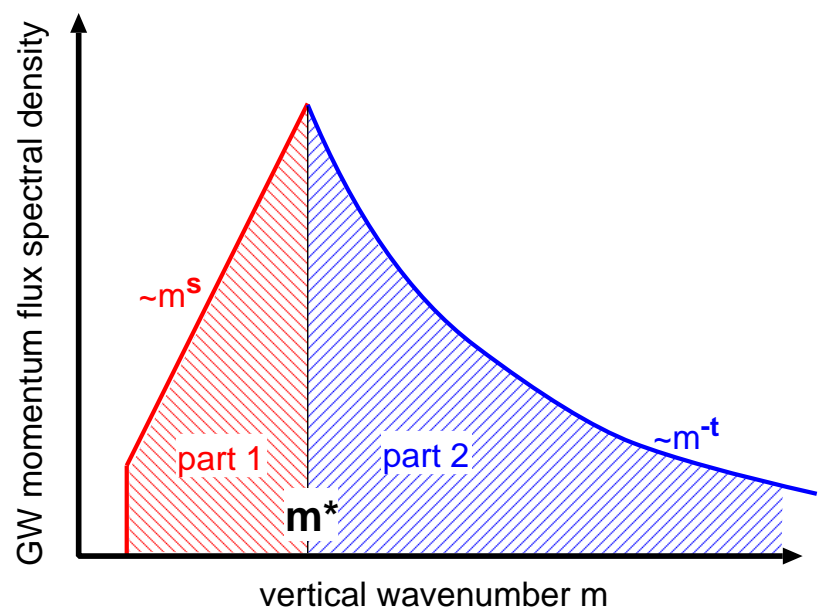

Fig. 1. GW momentum flux vertical wavenumber spectrum used in the Warner and McIntyre GW scheme at the model launch level. The spectrum consists of two parts: the unsaturated part (part 1, $m<m^{*}$ ) is $\sim m^{s}$, the saturated part (part 2, $m>m^{*}$ ) is $\sim m^{-t}$.

cent, 1995; Hertzog et al., 2001). On the other hand, the unsaturated part of the vertical wavenumber spectrum (at small vertical wavenumbers $m$ ) is not well defined observationally or theoretically (Fritts and Alexander, 2003).

For the characteristic wavenumber $m^{*}$ separating the saturated from the unsaturated part of the vertical wavenumber spectrum typical values of about $0.2-0.5$ cycles $/ \mathrm{km}$ have been reported for the lower stratosphere (Allen and Vincent, 1995; Hertzog et al., 2001; Tsuda and Hocke, 2002). However, the values given are subject to larger uncertainties mainly due to the required detrending of the vertical profiles of observations. In addition, the meteorological conditions at the measurement locations play an important role.

All these assumptions and observations are incorporated in the GW parameterization schemes used in GCMs. In the Warner and McIntyre GW parameterization scheme (Warner and McIntyre, 1996, 1999, 2001) for simplification the vertical wavenumber $(m)$ launch spectrum for gravity wave momentum flux (GW-MF) is divided into two parts: The unsaturated part at low vertical wavenumbers $m$ is assumed to increase with $m^{s}$ (with a small- $m$ cutoff value $m_{\text {cut }}$ ). In Warner and McIntyre (2001) a standard value of $s=1$ is used. However, this spectral slope is not very well defined by observations or theoretically (Fritts and Alexander, 2003). The saturated part of the spectrum at high vertical wavenumbers is assumed to decline with $m^{-t}$ with the standard value $t=3$. The characteristic wavenumber $m^{*}$ separates the unsaturated from the saturated spectral part (see Fig. 1).

Another parameter $\beta$ defines the value of the GW energy density $E_{0}$ at the launch level:

$$
E_{0}=\beta N^{2} / m^{* 2}
$$

where $N$ is the buoyancy frequency. In the Warner and McIntyre scheme the value of $\beta$ is proportional to the amount of GW-MF (at all altitudes) and the GW drag derived from it. From theoretical assumptions the value of $\beta$ is about 0.1 with an uncertainty of about a factor of two (Warner and McIntyre, 1996; Fritts and Alexander, 2003). Since $\beta$ in the way it is used in the Warner and McIntyre GW parameterization scheme simply scales the values of GW-MF and gravity wave drag without changing relative structures of the overall distributions this value of $\beta$ will be used for all calculations shown in this manuscript (see Sects. 3, 4 and 5).

The choice of these launch parameters is based more on theoretical assumptions than on observations (Warner and McIntyre, 1996, 1999, 2001). This is why normally fixed parameter values are taken for all longitudes and latitudes, and global variations of these values due to different sources and mechanisms exciting GWs remain out of consideration. This means the Warner and McIntyre GW scheme (like all other general GW parameterization schemes) will describe only the global background distribution of GWs.

In particular, orographically excited GWs (mountain waves) and GWs excited by deep convection, mainly in the tropics and subtropics, are not covered by these general GW parameterization schemes. GWs excited by those processes have to be described by separate models (McFarlane, 1987; Eckermann et al., 2000; Chun and Baik, 1998, 2002; Beres et al., 2005).

Global data sets of observed GW-MF can help to remedy the lack of experimental constraints. It can be tested whether the simplifying assumptions mentioned above are justified and, in particular, whether a GW parameterization scheme with a selected set of fixed model parameters is able to reproduce the observed horizontal and vertical patterns of GWMF.

This comparison has to be made in terms of momentum flux rather than comparing potential wave energy. The scale separation approach (cf. Sect. 3 and Appendix A) to isolate GWs from other atmospheric fluctuations retains also inertio GWs of very long horizontal wavelengths. These waves predominately exist at the equator (Alexander et al., 2002), but can spread to higher latitudes with increasing altitudes (Preusse et al., 2006) propagating several $1000 \mathrm{~km}$ in the horizontal. These waves cannot be described by a model assuming mid frequency approximation and purely vertical wave propagation. Though these waves are contributing a large part of the measured wave potential energy they contribute little to the measured momentum flux (Ern et al., 2004; Preusse et al., 2006) and they are badly represented in the parameterization scheme. Comparing momentum flux, hence makes model and measurement comparable at all and, in addition, strengthens the scale separation approach by focusing on shorter horizontal wavelengths less likely influenced by non-GW signatures (cf. Appendix A).

The only true global experimental data set of GW-MF available was derived from temperature altitude profiles 
measured by the CRyogenic Infrared Spectrometers and Telescopes for the Atmosphere (CRISTA) instrument (Ern et al., 2004). Therefore the purpose of this paper is to compare GW-MF distributions from both CRISTA flights with simulated distributions from the Warner and McIntyre GW parameterization scheme and by variation of the free parameters to infer constraints for the model launch parameters.

\section{CRISTA gravity wave momentum flux}

\subsection{Algorithm}

The CRISTA instrument was part of two Space Shuttle missions in November 1994 (CRISTA-1) and August 1997 (CRISTA-2). A high-resolution measuring grid in all three spatial dimensions was obtained by using three telescopes simultaneously and by cooling the instrument with supercritical helium to improve the measurement speed (Offermann et al., 1999). Atmospheric temperatures were derived from $\mathrm{CO}_{2}$ infrared limb emissions at $12.6 \mu \mathrm{m}$ (Riese et al., 1999).

To separate temperature fluctuations due to GWs from larger scale atmospheric structures like planetary waves, the temperature data were detrended using a zonal wavenumber 0-6 Kalman filter. The so-obtained vertical profiles of residual temperatures were analyzed for GWs using a combination of maximum entropy method and harmonic analysis and vertical profiles of GW amplitudes, vertical wavelengths and phases of the two strongest vertical wave components were derived (Preusse et al., 2002).

The short horizontal sampling distance of about $200 \mathrm{~km}$ along the satellite track is just sufficient to estimate the horizontal wavelengths of the GWs from GW phase differences between pairs of consecutive altitude profiles. Based on the determined GW temperature amplitudes, vertical and horizontal wavelengths it was possible to derive absolute values of GW-MF from satellite data for the first time (Ern et al., 2004).

Only absolute values of GW-MF (i.e., not the direction of GW-MF) could be derived due to limitations of the horizontal sampling. To derive vectors of GW-MF a high-resolution 2D horizontal sampling of about $40 \mathrm{~km}$ along and about $40 \mathrm{~km}$ across the satellite track is needed (Riese et al., 2005).

Another problem when determining GW-MF are aliasing effects: The limb scanning geometry allows CRISTA to detect GWs with horizontal wavelengths as short as $\sim 100 \mathrm{~km}$ (Preusse et al., 2002; Ern et al., 2005). This is much shorter than the limiting wavelength (Nyquist wavelength) of $400 \mathrm{~km}$ (i.e. twice the horizontal sampling distance along the satellite track) that can be resolved unambiguously by the CRISTA horizontal sampling. Therefore limitations arising from the CRISTA sampling are more severe than those arising from the limb scanning geometry.

This undersampling of GWs causes aliasing effects: horizontal wavelengths determined for the fraction of undersam- pled waves are systematically too long and the GW-MF carried by these waves is underestimated. To compensate for this low-bias in CRISTA GW-MF an aliasing correction has been applied. Of course, such an empirical correction is subject to large errors. The correction is based on the mean horizontal wavelength in regions of $30^{\circ}$ longitude times $20^{\circ}$ latitude (Ern et al., 2004) and generally increases the CRISTA GW-MF values. The average correction is about a factor of 1.7 and the correction is limited to not more than a factor of 2. Largest corrections are made at high northern and high southern latitudes. Hence, the contrast between equatorial and polar latitudes is enhanced. For a more detailed description of the GW-MF algorithm see Ern et al. (2004).

The single-wave spectral results from CRISTA altitude profiles will be compared with results from a spectral GW parameterization. Since a spectral model reflects the GW mean state at a given location we have to average over the single CRISTA profiles to obtain comparable values. Therefore for all further analyses the GW-MF values determined for the abovementioned regions of $30^{\circ}$ longitude times $20^{\circ}$ latitude will be used. Averaging the CRISTA profiles has also the advantage of reducing the scatter due to intermittent GW sources inherent in the single CRISTA profiles. In addition, aliasing corrected GW-MF values can be used.

\subsection{GW-MF during CRISTA-1 and CRISTA-2}

There are some differences between the CRISTA-1 (November 1994) and CRISTA-2 (August 1997) data sets with respect to GW analysis, derivation of GW-MF, and the interpretation of the results.

First, the meteorological conditions during the two CRISTA missions were different. Latitude altitude cross sections of the zonal mean zonal wind for the CRISTA-1 and CRISTA-2 missions are shown in Fig. 2. During CRISTA-2 there is a wind reversal in the northern hemisphere already at low altitudes between 20 and $25 \mathrm{~km}$ (see Fig. 2b).

This wind reversal prevents mountain waves from propagating towards higher altitudes. In the southern hemisphere there is no wind reversal and mountain waves can propagate through the whole stratosphere. However, there are only few major mountain ridges in the southern hemisphere. Therefore orographic GWs should not be a dominant effect. Since nonorographic GW parameterization schemes (e.g., the Warner and McIntyre GW parameterization scheme) do not cover contributions due to mountain waves the CRISTA2 data set of GW-MF should be suited better for comparisons with those models than, for example, the CRISTA-1 GW-MF data. During CRISTA-1 there is no wind reversal in the northern hemisphere and the wind reversal observed in the southern hemisphere is at higher altitudes (about 20 $35 \mathrm{~km}$ depending on latitude, see Fig. 2a). Therefore part of the GWs observed during CRISTA- 1 are mountain waves (see also Eckermann and Preusse, 1999; Preusse et al., 2002; Jiang et al., 2004a). 
(a) CRISTA-1 (Nov. 94)

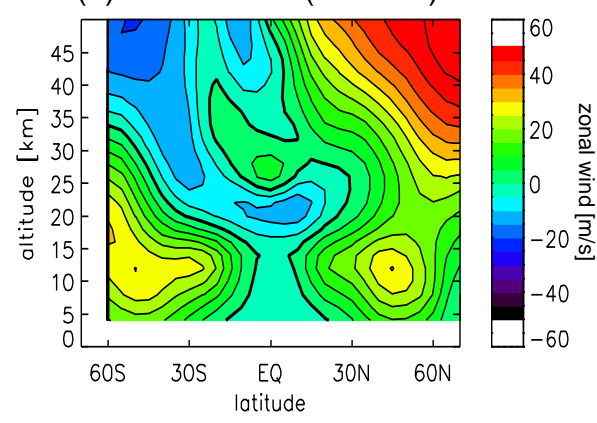

(b) CRISTA-2 (Aug. 97)

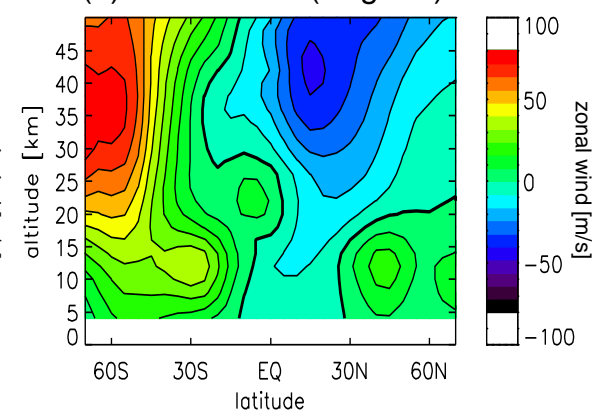

Fig. 2. Zonal mean zonal wind for CRISTA-1 (a) and CRISTA-2 (b) calculated from UKMO data interpolated to CRISTA measurement times and locations. Zero wind is represented by a bold contour line. Please note that the color code is different in (a) and (b).

In addition, the CRISTA-2 GW-MF data should generally be better suited for global comparisons due to the observed strong meridional variation of GW-MF with very high values in the region of the southern polar jet (Ern et al., 2004), providing a high-contrast distribution of GW-MF. This meridional variation is less pronounced in the CRISTA-1 GW-MF data, see also Sect. 3. Due to differences between the measurement modes of the two CRISTA missions the CRISTA-2 values of GW-MF also cover a larger altitude interval (about 20-50 km) compared to CRISTA-1 (about 20-40 km).

\section{Horizontal distributions of CRISTA GW-MF com- pared to standard Warner and McIntyre scheme re- sults}

Previous investigations have shown that good agreement between the GW-MF horizontal distributions at $25 \mathrm{~km}$ altitude obtained from CRISTA-2 and the Warner and McIntyre scheme using the standard set of launch parameters $(s=1$, $\lambda_{z, \text { launch }}^{*}=2 \pi / m_{\text {launch }}^{*}=2 \mathrm{~km}$ ) can only be achieved if a low model GW launch level is chosen (Ern et al., 2004, 2005). It should be mentioned that we always use the standard value $\beta \approx 0.1$ without loss of generality since $\beta$ in the Warner and McIntyre GW parameterization scheme simply scales the GW-MF values without changing the relative distributions (see Sect. 1).

Using higher launch levels usually results in model GWMF distributions too symmetric with respect to the equator and the longitudinal structure of GW-MF is not reproduced properly (Ern et al., 2004, 2005). In this section some examples are shown for both CRISTA-1 and CRISTA-2 and a low model launch level of $464 \mathrm{mbar}$ (about $5.4 \mathrm{~km}$ ).

Figure 3 shows horizontal distributions of GW-MF absolute values for CRISTA-2 at altitudes of $25 \mathrm{~km}$ (Fig. 3a), $35 \mathrm{~km}$ (Fig. 3c), and $45 \mathrm{~km}$ (Fig. 3e). Also shown are results of GW-MF absolute values calculated with the Warner and McIntyre GW parameterization scheme using meteorological data (temperature and wind fields) from the UK Met
Office (UKMO) stratosphere-troposphere assimilation system (Swinbank and O'Neill, 1994) interpolated to CRISTA2 measurement times and locations for the same altitudes (25 km (Fig. 3b), $35 \mathrm{~km}$ (Fig. 3d), and $45 \mathrm{~km}$ (Fig. 3f)). The model GW-MF results are filtered according to the horizontal and vertical wavelengths of GWs visible for the CRISTA instrument: CRISTA is able to detect waves with horizontal wavelengths from $\sim 100 \mathrm{~km}$ to $\sim 5000 \mathrm{~km}$ and vertical wavelengths in the intervals $\lambda_{z} \in[5 \mathrm{~km}, 25 \mathrm{~km}]$ (CRISTA-1) and $\lambda_{z} \in[6 \mathrm{~km}, 30 \mathrm{~km}]$ (CRISTA-2) (Ern et al., 2005).

Obviously, the good agreement between the horizontal relative structures of both CRISTA-2 and model data found earlier by Ern et al. (2004) at $25 \mathrm{~km}$ altitude is also valid for the other altitudes. There are some differences in details of the horizontal structures. For example, the very high values of GW-MF found in the CRISTA-2 data over the Antarctic Peninsula and the southern tip of South America, or the high values over Southeast Asia and the Gulf of Mexico, are all underrepresented in the model results and indicate localized GW sources in these regions. On the other hand, there is slightly too much GW-MF in the northernmost latitudes of the model results, especially at $45 \mathrm{~km}$ altitude. It should also be mentioned that the color scales of Figs. 3a-f are all different to allow the comparison of horizontal structures (see contour labels). At $25 \mathrm{~km}$ altitude the model values of GW-MF are considerably lower than the CRISTA- 2 values by over a factor of 5 (global average), whereas at $45 \mathrm{~km}$ altitude the model values are lower than the CRISTA- 2 values by not more than a factor of about 2 (global average).

Figure 4 shows a comparison between CRISTA-1 GW-MF absolute values and Warner and McIntyre results like the one in Fig. 3 with the same model launch parameters (launch level $464 \mathrm{mbar}, s=1$, and $\lambda_{z}^{*}$, launch $=2 \mathrm{~km}$ ). Since the altitude range of the CRISTA-1 GW-MF data is smaller only altitudes of $25 \mathrm{~km}$ and $35 \mathrm{~km}$ are shown for the CRISTA-1 (Figs. 4a, c) and the model data (Figs. 4b, d). Again, the color scales are different in Figs. 4a-d to highlight features of the horizontal distributions. 

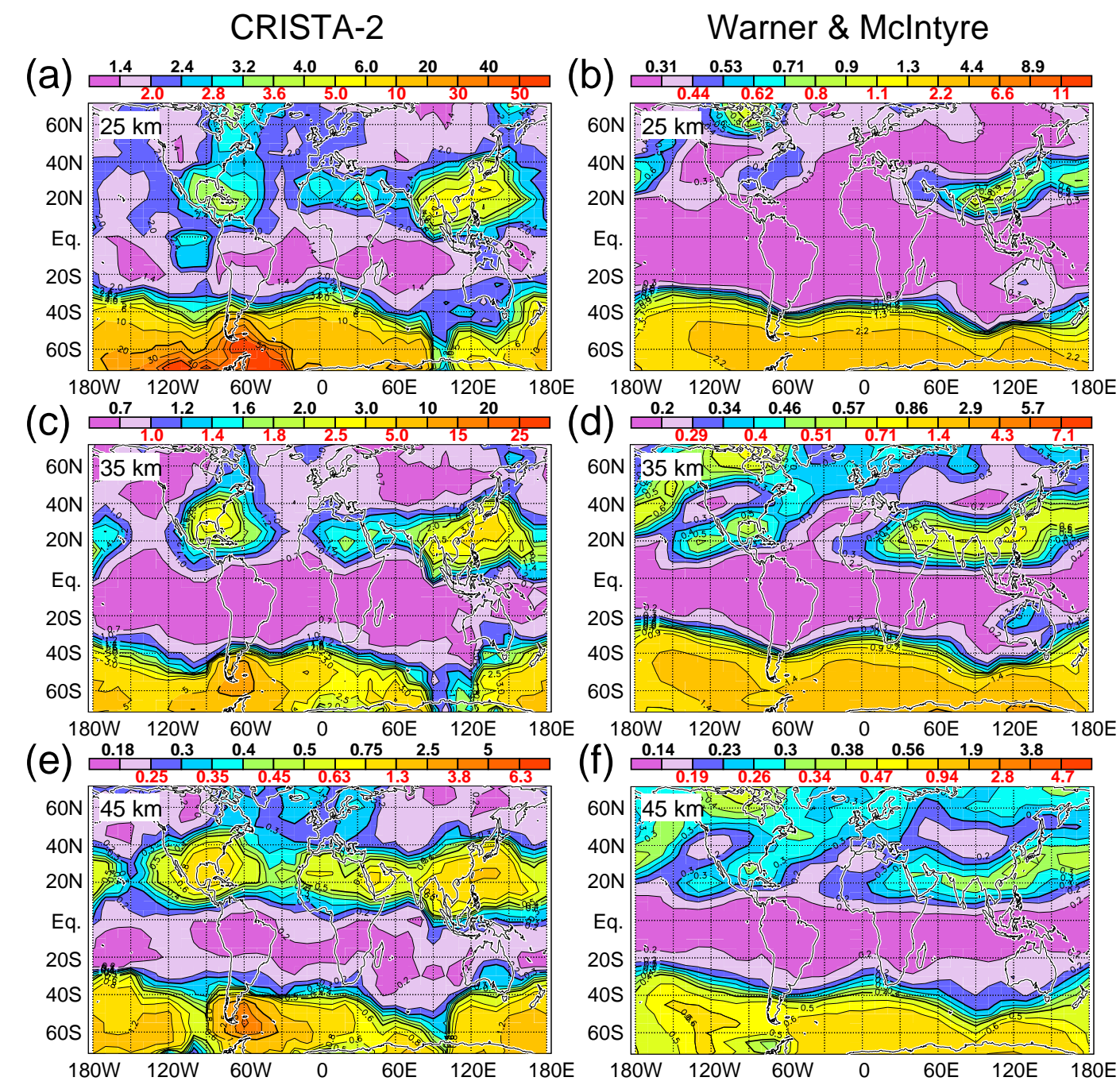

Fig. 3. Shown are horizontal distributions of GW-MF absolute values in $\mathrm{mPa}$ (see color bars and contour labels) for CRISTA-2 (August 1997) at altitudes of $25 \mathrm{~km}$ (a), $35 \mathrm{~km}$ (c), and $45 \mathrm{~km}$ (e). Also shown are horizontal distributions of GW-MF absolute values in mPa calculated with the Warner and McIntyre GW parameterization scheme at the same altitudes $(25 \mathrm{~km}(\mathbf{b}), 35 \mathrm{~km}(\mathbf{d})$, and $45 \mathrm{~km}(\mathbf{f}))$ for a fixed model GW launch level at 464 mbar (about $5.4 \mathrm{~km}$ ) and the spectral parameters $s=1$ and $\lambda_{z \text {, launch }}^{*}=2 \mathrm{~km}$. Instrumental visibility filtering has been applied to the model values. Please note that due to differences in the GW-MF absolute values contour lines and color codes are different at different altitudes and also different for CRISTA and model results.

Figure 3a was reproduced from Fig. 3d in: Ern, M., Preusse, P., Alexander, M. J., and Warner, C. D., Absolute values of gravity wave momentum flux derived from satellite data, J. Geophys. Res., 109, D20103, doi:10.1029/2004JD004752, 2004. Copyright [2004] American Geophysical Union. Reproduced by permission of American Geophysical Union.

The agreement between model and CRISTA-1 horizontal relative structures of GW-MF absolute values is worse than in the CRISTA- 2 case. The agreement improves with altitude, however, in the $25 \mathrm{~km}$ as well as in the $35 \mathrm{~km}$ CRISTA-1 maps there are additional regions of high GW-MF over entire South America which are not present in the model maps. In addition, in the model maps there are high values of GW-MF at the southernmost latitudes, which are not present in the CRISTA-1 data. Also the bands of high GW-MF in northern latitudes are too uniform and too pronounced in the model data, especially at $25 \mathrm{~km}$ altitude.
Like for the CRISTA-2 data, the GW-MF absolute values from CRISTA-1 are considerably higher than the model results. At $25 \mathrm{~km}$ altitude they are higher by a factor of about 5 (global average), and at $35 \mathrm{~km}$ altitude the CRISTA- 1 values are higher by a factor of about 3 (global average).

As a criterion for the agreement between measured and modeled horizontal distribution a correlation coefficient can be calculated grid point by grid point from the horizontal maps. Correlation will be especially high if the modeled GW-MF exhibits the same meridional asymmetry as the 

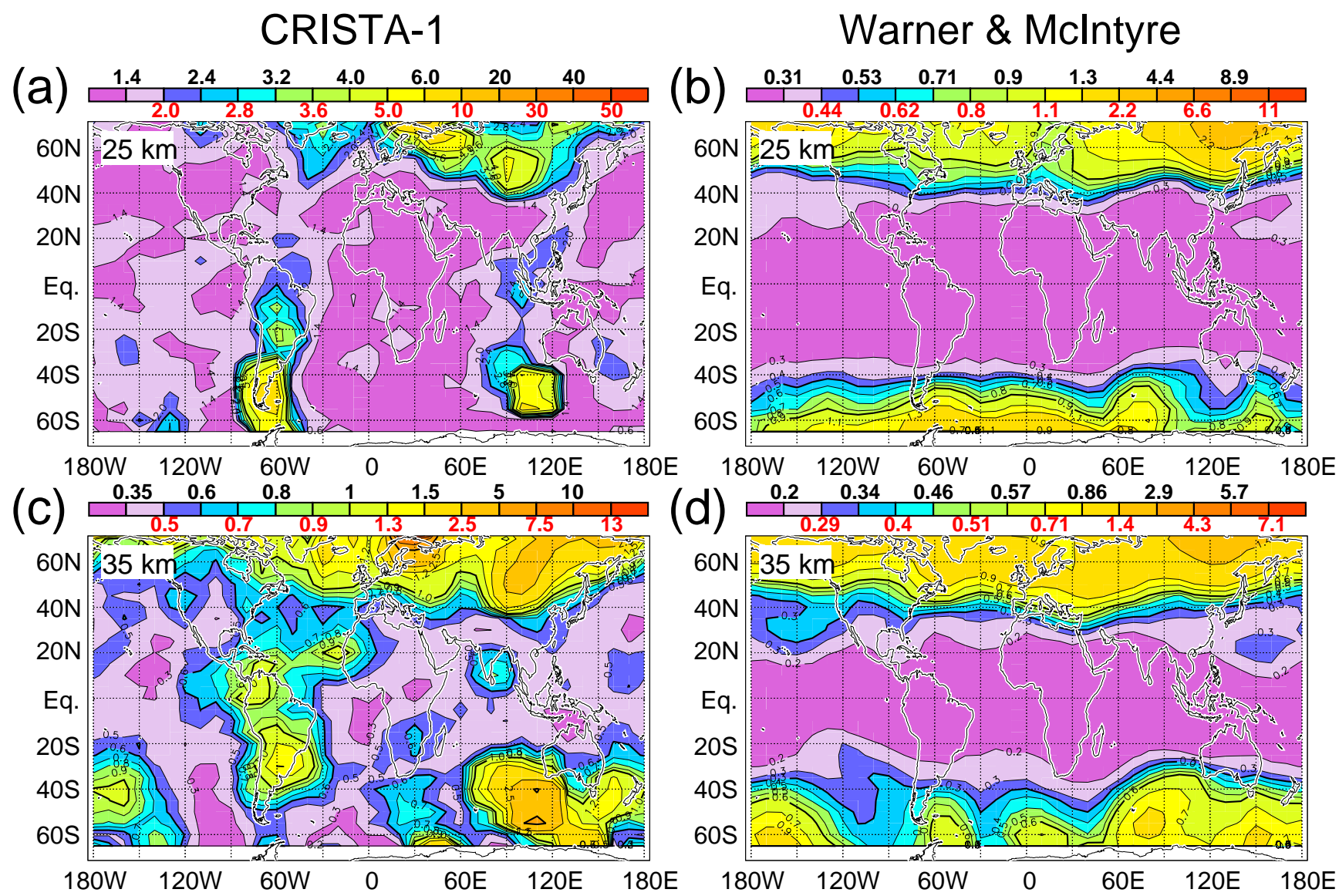

Fig. 4. Horizontal distributions of GW-MF absolute values in $\mathrm{mPa}$ (see color bars and contour labels) for CRISTA-1 (November 1994) at altitudes of $25 \mathrm{~km}$ (a) and $35 \mathrm{~km}$ (c). Also shown are horizontal distributions of GW-MF absolute values in mPa calculated with the Warner and McIntyre GW parameterization scheme at the same altitudes $(25 \mathrm{~km} \mathrm{(b),} 35 \mathrm{~km}$ (d)) for a fixed model GW launch level at $464 \mathrm{mbar}$ about $5.4 \mathrm{~km}$ ) and the spectral parameters $s=1$ and $\lambda_{z \text { launch }}^{*}=2 \mathrm{~km}$. Instrumental visibility filtering has been applied to the model values. Please note that due to differences in the GW-MF absolute values contour lines and color codes are different at different altitudes and also different for CRISTA and model results.

CRISTA GW-MF and if longitudinal variations are the same. The correlation between horizontal distributions will be used to evaluate choices of model parameters in Sect. 4.

\section{Comparison of horizontal GW momentum flux distri- butions for different choices of launch level, $\lambda_{z}^{*}$, launch and $s$}

Given the horizontal distributions of GW-MF from CRISTA as reference possible ranges of the GW launch parameters used in the Warner and McIntyre scheme can be determined. Especially the launch altitude and the spectral parameters $\lambda_{z, \text { launch }}^{*}\left(\lambda_{z, \text { launch }}^{*}=2 \pi / m_{\text {launch }}^{*}\right)$ and $s$ are poorly constrained by measurements. Therefore we will focus on these parameters in the following.
Other parameters like the spectral slopes $t=3$ (saturated part of vertical wavelength spectrum assumed to decrease with $m^{-3}$ ) and $p=5 / 3$ (intrinsic frequency $\hat{\omega}$ spectrum of the GW wave energy density assumed to decrease with $\hat{\omega}^{-5 / 3}$ ) are better constrained by observations (see Sect. 1) and will be left unchanged.

It should be stated clearly that the error ranges of CRISTA GW-MF and Warner and McIntyre model results are quite large (Ern et al., 2004). Therefore deviations between CRISTA and model GW-MF absolute values will be inside the error range if deviations are less than a factor of about 4-5. (Relative structures are subject to much smaller errors since several error sources shift the distribution in total (Ern et al., 2004).) Nevertheless, it makes sense to compare CRISTA and Warner and McIntyre model results for different choices of model parameters to quantify the sensitivity on the different parameters, and to find out whether there is 

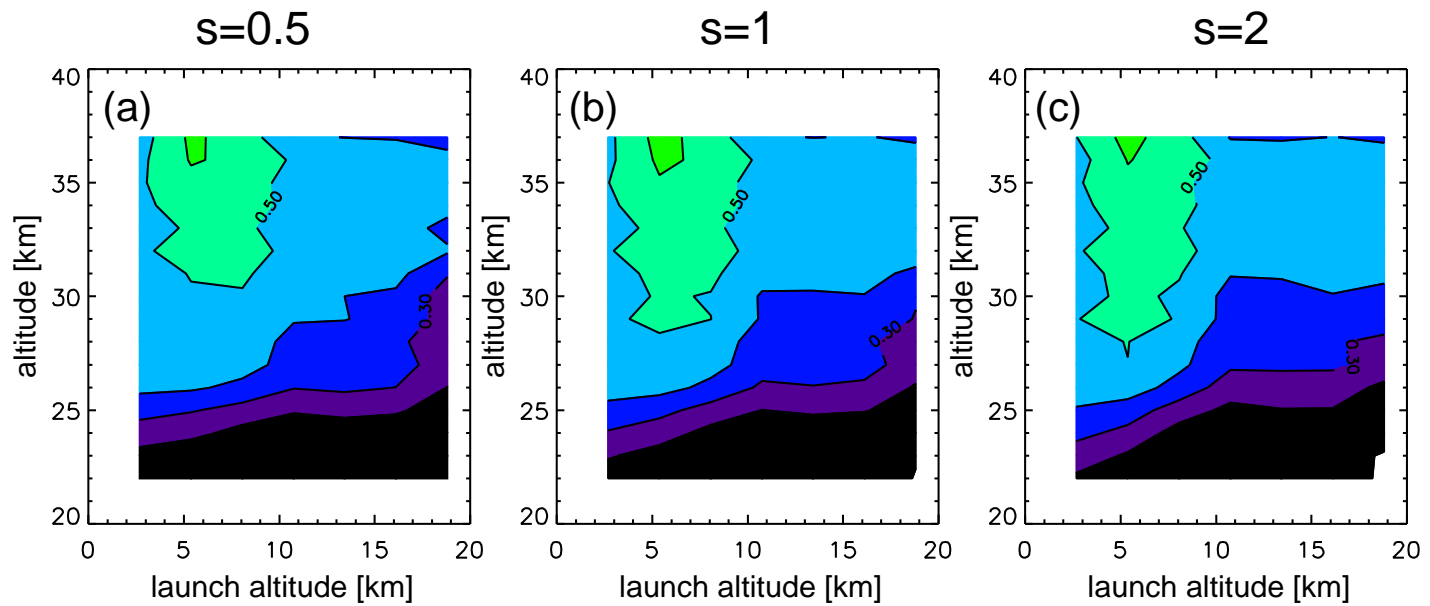

Fig. 5. Shown are for the Warner and McIntyre/CRISTA-1 comparison of horizontal GW-MF distributions correlation coefficients versus altitude and model launch altitude for the choice of $\lambda_{z, \text { launch }}^{*}=2 \mathrm{~km}$ and $s=0.5$ (a), $s=1$ (b), and $s=2.0$ (c). Contour lines are: $0.2,0.3,0.4$, $0.5,0.6$.
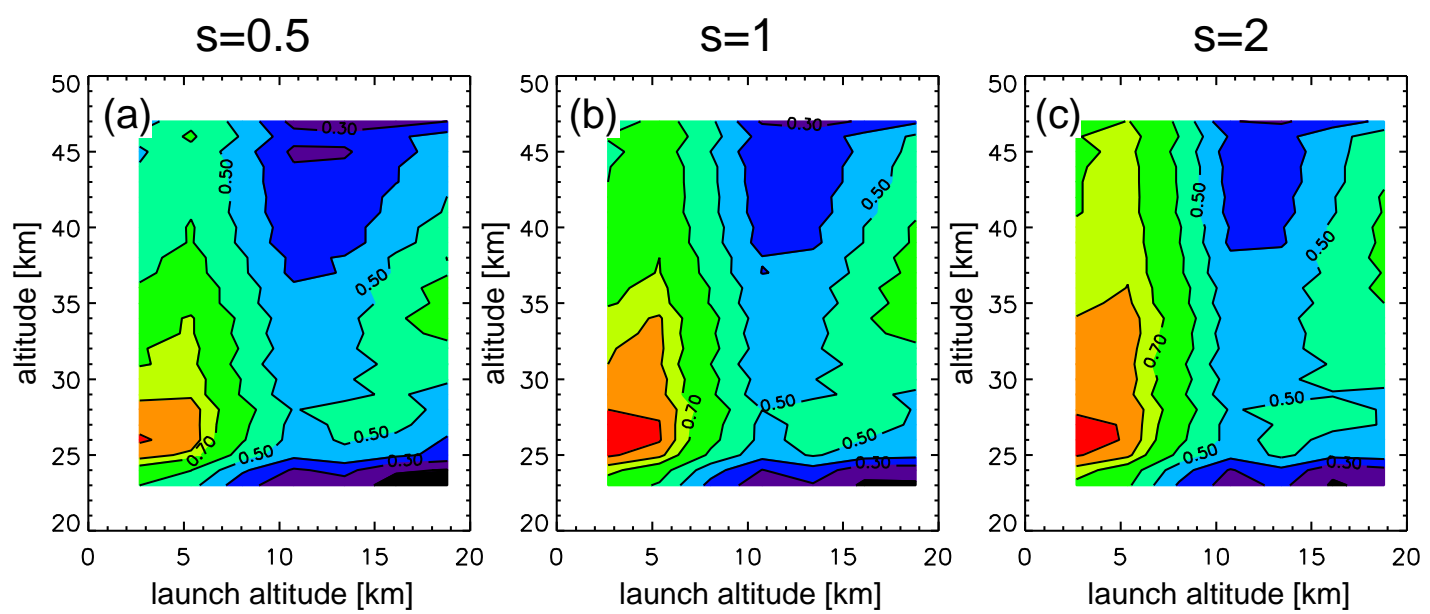

Fig. 6. Same as Fig. 5, but for CRISTA-2. Contour lines are: $0.2,0.3,0.4,0.5,0.6,0.7,0.75,0.8$.

an optimal set of parameters. This optimal set of parameters would have to fulfill two main criteria: high correlation of the horizontal distributions and comparable values of GW-MF.

It should also be noted that the CRISTA data sets are only about one week of data each and the results obtained in this paper are based on this limited data set only. This means some differences in the results could occur in other seasons and if meteorological conditions are different. However, there is evidence that the GW activity in August is similar in different years (Jiang et al., 2004b; Preusse et al., 2004; Preusse et al., 2006).

In addition, CRISTA GW-MF is available only in limited altitude regions of about $20-40 \mathrm{~km}$ for CRISTA-1 and 20$50 \mathrm{~km}$ for CRISTA-2. This means the altitudes around the mesopause where wind accelerations are maximum are not covered by the GW-MF data available.

4.1 Determination of an optimum model launch altitude by variation of $s$ and $\lambda_{z \text {, launch }}^{*}$

For the standard choice $\lambda_{z \text {,launch }}^{*}=2 \mathrm{~km}$ Figs. 5 and 6 show contour plots of the correlation coefficient between horizontal distributions of GW-MF of the Warner and McIntyre model and CRISTA as a reference. The results were obtained for the altitude range of available CRISTA GW-MF and seven different model launch altitudes from about $2.7 \mathrm{~km}$ to about $19 \mathrm{~km}$ (according to the UKMO pressure levels from 681 to 68.1 mbar). 

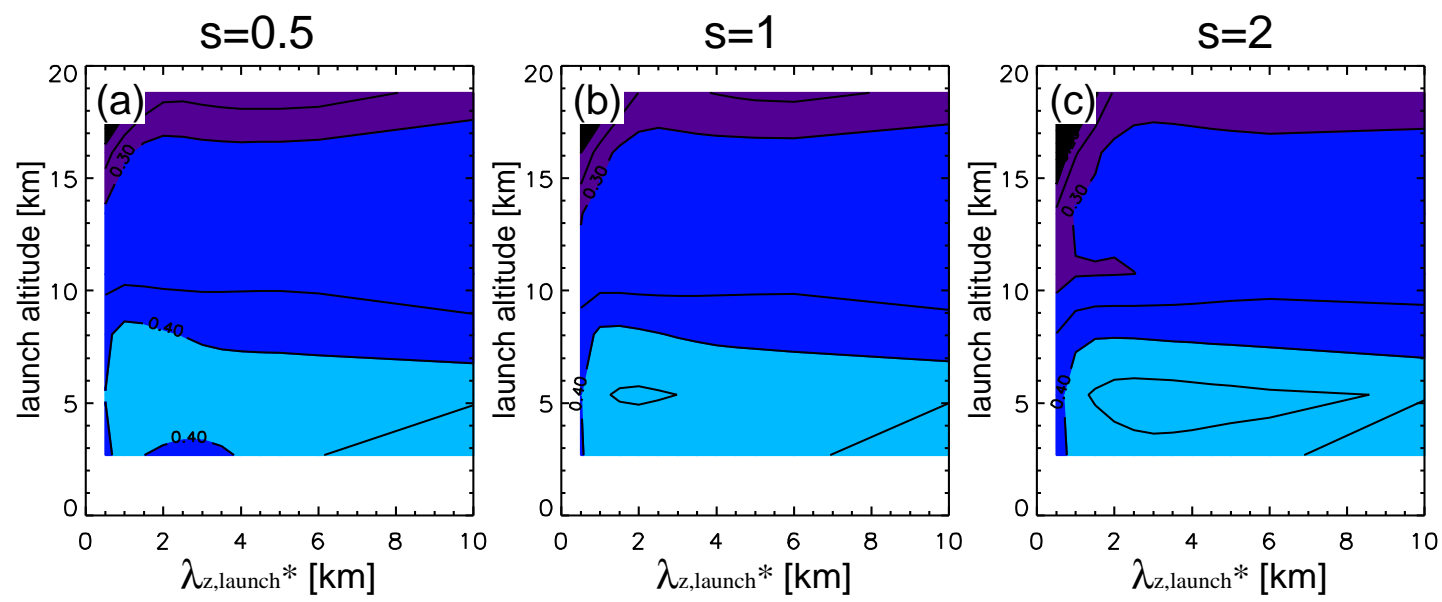

Fig. 7. Warner and McIntyre/CRISTA-1 comparison of horizontal GW-MF. Correlation coefficients as shown in Fig. 5 are averaged over the whole altitude range $22-37 \mathrm{~km}$. Varied are model launch altitude and $\lambda_{z \text {, launch }}^{*}$ for $s=0.5$ (a), $s=1$ (b), and $s=2.0$ (c).

I.e., the correlation coefficients of Figs. $5 \mathrm{a}-\mathrm{c}$ averaged over the whole altitude range are the column at $\lambda_{z}^{*}$, launch $=2 \mathrm{~km}$ in Figs. $7 \mathrm{a}-\mathrm{c}$. Contour lines are: $0.2,0.25,0.3,0.35,0.4,0.45$.
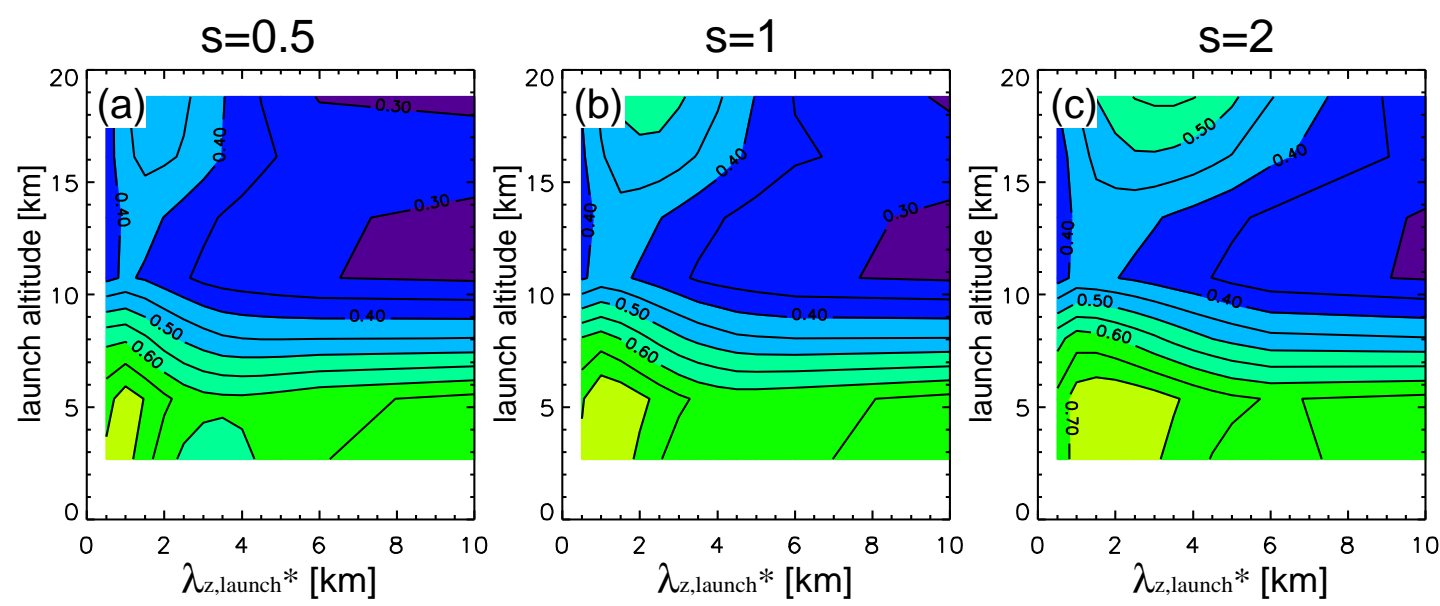

Fig. 8. Warner and McIntyre / CRISTA-2 comparison of horizontal GW-MF. Correlation coefficients as shown in Fig. 6 are averaged over the whole altitude range $23-47 \mathrm{~km}$. Varied are model launch altitude and $\lambda_{z \text {, launch }}^{*}$ for $s=0.5$ (a), $s=1$ (b), and $s=2.0$ (c).

I.e., the correlation coefficients of Figs. $6 \mathrm{a}-\mathrm{c}$ averaged over the whole altitude range are the column at $\lambda_{z, \text { launch }}^{*}=2 \mathrm{~km}$ in Figs. $8 \mathrm{a}-\mathrm{c}$. Contour lines are: $0.2,0.25,0.3,0.35,0.4,0.45,0.5,0.55,0.6,0.65,0.7$.

Figure 5 shows the comparison with CRISTA- 1 and Fig. 6 the comparison with CRISTA-2 GW-MF, respectively. Correlation coefficients are given for the spectral launch parameters $s=0.5$ (a), $s=1$ (b), and $s=2$ (c). As can be seen from Figs. 5 and 6 for all choices of $s$ : considering the whole range of measurement altitudes the correlation is highest for the second lowest launch level 464 mbar (launch latitude $\sim 5.4 \mathrm{~km}$ ). It is also very high for the lowest launch level 681 mbar (launch latitude $\sim 2.7 \mathrm{~km}$ ). Since this result is similar also for other choices of launch parameters $\lambda_{z, \text { launch }}^{*}$ and $s$ (not shown) we average the correlation coefficients over all altitudes for further comparisons.

Results based on this vertical averaging are shown in Figs. 7 and 8 for CRISTA- 1 and CRISTA-2, respectively. Figures $7 \mathrm{a}-\mathrm{c}$ and $8 \mathrm{a}-\mathrm{c}$ show contour plots of the correlation coefficient averaged over the whole altitude range for different launch levels and different values of $\lambda_{z, \text { launch. Figures } 7}^{*}$ and 8 show the results for the choice (a) $s=0.5$, (b) $s=1$, and (c) $s=2$. Again, from these figures it can be seen clearly that the correlation is highest for the lowest two launch levels 681 


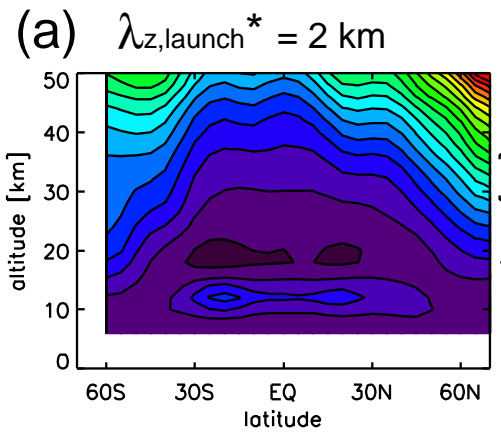

(b) $\lambda_{z, \text { launch }}{ }^{*}=4 \mathrm{~km}$

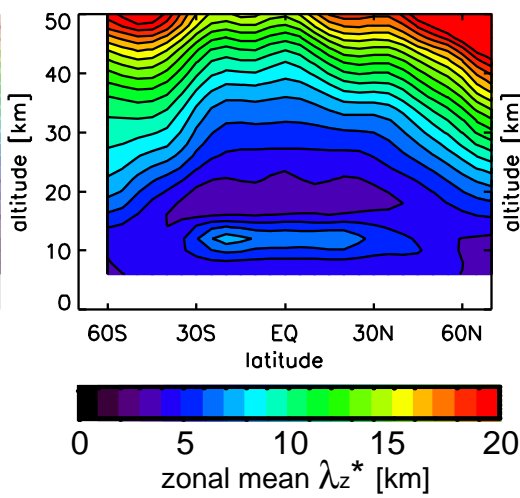

(C) $\lambda_{z, \text { launch }^{*}}=6 \mathrm{~km}$

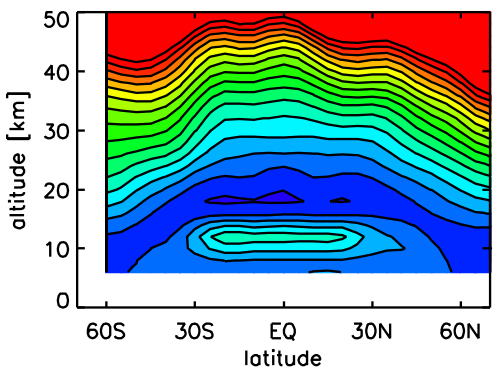

Fig. 9. Zonal mean cross section of $\lambda_{\mathrm{z}}^{*}$ obtained from the Warner and McIntyre scheme (launch level $464 \mathrm{mbar}, s=1$ ) for the direction of maximum GW-MF during CRISTA-1 (no observational filter applied) and $\lambda_{z, \text { launch }}^{*}=2 \mathrm{~km}(\mathbf{a}), \lambda_{z, \text { launch }}^{*}=4 \mathrm{~km}(\mathbf{b})$, and $\lambda_{z, \text { launch }}^{*}=6 \mathrm{~km}(\mathbf{c})$.

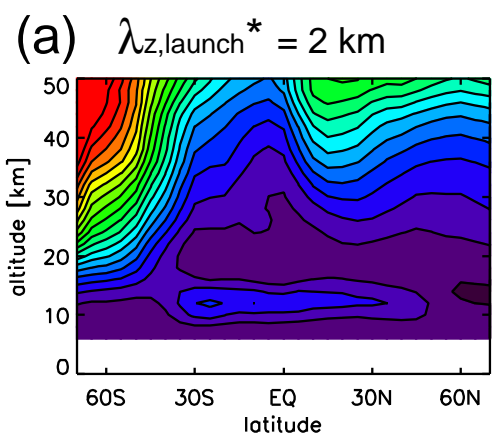

(b) $\lambda_{z, \text { launch }}{ }^{*}=4 \mathrm{~km}$

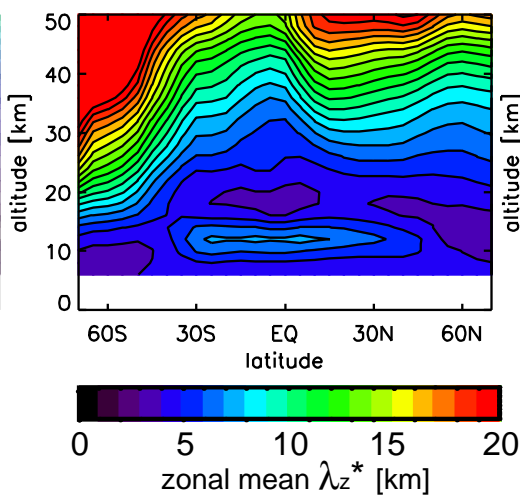

(C) $\lambda_{z, \text { launch }^{*}}=6 \mathrm{~km}$

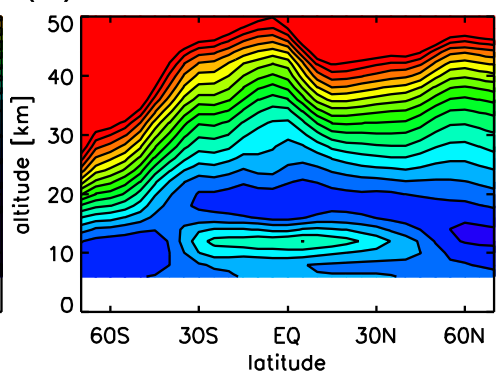

Fig. 10. Same as Fig. 9 but for Warner and McIntyre model GW-MF during CRISTA-2.

and 464 mbar ( $\sim 2.7$ and $\sim 5.4 \mathrm{~km})$ with the 464 mbar launch level giving the best results.

This means that the launch level $464 \mathrm{mbar}(\sim 5.4 \mathrm{~km})$ is the best choice for both CRISTA-1 and CRISTA-2 and is some kind of compromise for a globally fixed value of the launch altitude. This is valid for almost all choices of $\lambda_{z, \text { launch }}^{*}$ and $s$. Consequently, this launch level will be used for all following investigations.

\subsection{Altitude dependence of $\lambda_{z}^{*}$}

To determine the correct value of $\lambda_{z \text {,launch }}^{*}=2 \pi / m_{\text {launch }}^{*}$ direct comparisons with measurements of the spectral shape of the GW vertical wavenumber spectrum would be highly desirable. Figure 9 shows latitude altitude cross sections of zonal mean $\lambda_{z}^{*}$ for the CRISTA-1 period (November 1994) obtained from the Warner and McIntyre scheme with the GW launch parameters $s=1$, launch level $464 \mathrm{mbar}(\sim 5.4 \mathrm{~km})$ and the choices $\lambda_{z \text {, launch }}^{*}=2 \mathrm{~km}(\mathrm{a}), \lambda_{z \text {, launch }}^{*}=4 \mathrm{~km}(\mathrm{~b})$, and $\lambda_{z, \text { launch }}^{*}=6 \mathrm{~km}(\mathrm{c})$. Figure 10 shows the same, but for the CRISTA-2 period (August 1997).

The Warner and McIntyre scheme calculates values $m_{i}{ }^{*}$ for all directions $i$, in which GW-MF is derived. In this paper always 4 directions are used (corresponding to the cardinal points). The values of $m^{*}$ used to calculate the values of $\lambda_{z}^{*}$ shown in Figs. 9 and 10 were obtained by calculating a mean of the single $m_{i} *$ components weighted by the squares of the associated GW-MF components without CRISTA observational filter applied. This means the distributions shown in Figs. 9 and 10 are about what an ideal instrument would be measuring if the Warner and McIntyre model output would be the "truth". However, it should be noted that, depending on the specific shape of the spectrum, the maximum of the GW-MF vertical wavenumber spectrum used in the Warner and McIntyre scheme can be located at vertical wavelengths somewhat larger than the values of $\lambda_{z}^{*}$ shown. 
From Figs. 9 and 10 can be seen that, starting from $\lambda_{z \text {,launch }}^{*}$ at the launch level $464 \mathrm{mbar}$, the value $\lambda_{z}^{*}$ basically increases with altitude. This well-known effect is caused by growth of the GW amplitudes with altitude, leading to an extension of the saturated part of the vertical wavenumber spectrum towards lower $m$ (longer vertical wavelengths) at higher altitudes (Fritts and VanZandt, 1993; Gardner, 1994). In addition, there are also meridional variations mainly caused by Doppler shift of the vertical wavenumber spectrum due to the vertical profile of the horizontal wind.

If the directions of horizontal wind and GW-MF are antiparallel the vertical wavenumber spectrum (this means also the location of $m^{*}$ ) is Doppler shifted towards lower values of $m$ (higher $\lambda_{z}$ ) and wave breaking is reduced. Therefore GW-MF for the GWs propagating opposite to the wind direction can be higher than GW-MF for GWs with zero Doppler shift. This explains why for CRISTA-2 GW-MF is enhanced inside the southern polar jet and also the values of high GWMF in the northern subtropics caused by subtropical easterlies. The opposite way around, the vertical wavenumber spectrum of GWs propagating parallel to the direction of the prevailing wind is shifted towards higher values of $m$. In this case wave breaking is stronger and GW-MF is strongly reduced. Therefore the prevailing propagation direction of GWs inside the polar jet is opposite to the wind direction.

It is a general feature of the $\lambda_{z}^{*}$ distributions shown in Figs. 9 and 10 that for low values of $\lambda_{z, \text { launch }}^{*}$ the average values of $\lambda_{z}^{*}$ are lower over the whole altitude range. In addition, the meridional structure of $\lambda_{z}^{*}$ is more pronounced for low

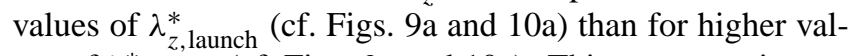
ues of $\lambda_{z \text {,launch }}^{*}$ (cf. Figs. 9c and 10c). This means an instrument with the capability to measure the vertical wavenumber spectrum from about $\lambda_{z}^{*} \lesssim 2 \mathrm{~km}$ up to $\lambda_{z}^{*} \gtrsim 20 \mathrm{~km}$ would be able to resolve the vertical $\lambda_{z}^{*}$ distribution and could give directly constraints to the launch value $\lambda_{z \text {, launch }}^{*}$ by a comparison with model data. However, up to date there is no experimental data set spanning such a wide interval of vertical wavelengths.

Some constraints can be inferred from the observations already mentioned in Sect. 1 (Allen and Vincent, 1995; Hertzog et al., 2001; Tsuda and Hocke, 2002). The experimental values of $m^{*}$ in the range of about $0.2-0.5$ cycles $/ \mathrm{km}$ (corresponding to $\lambda_{z}^{*}$ in the range $2-5 \mathrm{~km}$ ) are valid for the lower stratosphere and mainly from low and mid-latitudes. If these values are compared to Figs. 9 and 10 we can conclude that the model parameter $\lambda_{z \text {,launch }}^{*}$ should not exceed about $4 \mathrm{~km}$. Otherwise the model values of $\lambda_{z}^{*}$ would be too high in the lower stratosphere.

Further constraints of the parameter $\lambda_{z \text {,launch }}^{*}$ can be made by comparing measured and modeled distributions of GWMF. Therefore a comparison of GW-MF absolute values from CRISTA and the Warner and McIntyre scheme will be made in the following subsection to find possible ranges of $\lambda_{z \text {,launch }}^{*}$, and maybe to find even an optimum value for $\lambda_{z, \text { launch }}^{*}$
4.3 Influence of $\lambda_{z \text {,launch }}^{*}$ and $s$ on horizontal correlations and GW-MF absolute values

\subsubsection{Variation of $\lambda_{z, \text { launch }}^{*}$ and $s$}

From Sect. 3 we have seen that the standard choice of GW launch parameters $\left(s=1, \lambda_{z}^{*}\right.$, launch $\left.=2 \mathrm{~km}\right)$ used in the Warner and McIntyre scheme already provides good agreement with the horizontal structures found in CRISTA GW-MF absolute values. To find out whether this agreement can be further improved and whether the low-bias of model GWMF compared to CRISTA values can be reduced the values $\lambda_{z, \text { launch }}^{*}=2 \pi / m_{\text {launch }}^{*}$ and $s$ have been varied.

Figures 11a-c show deviations between horizontal distributions of GW-MF absolute values calculated with the Warner and McIntyre scheme and CRISTA-1 GW-MF as a reference. The deviations shown are the slopes of linear fits through the origin from scatter plots of model GW-MF vs. CRISTA GW-MF for every pair of horizontal maps. The reciprocal of the slopes has been taken for slopes $<1$ (at low $\lambda_{z \text {,launch }}^{*}$ ) to have the same color scale for GW-MF deviations in both directions. The logarithm of the GW-MF values has been used for the fits to avoid over-weighting of low values of GW-MF (see also Ern et al., 2004, 2005).

The Warner and McIntyre GW-MF was calculated for the launch level $464 \mathrm{mbar}$ and different altitudes and values $\lambda_{z, \text { launch }}^{*}=2 \pi / m_{\text {launch }}^{*}$.

Figure 11a is for $s=0.5$, (b) for $s=1$, and (c) for $s=2$. Low deviations (blue and purple colors) are found in the $\lambda_{z \text {,launch }}^{*}$ range of about $2-5 \mathrm{~km}$. Model values are lower than CRISTA-1 GW-MF to the left and higher to the right of the contour line labeled " 1 ". At values $\lambda_{z \text {,launch }}^{*}<1.5 \mathrm{~km}$ the model values are lower than the CRISTA- 1 values by over a factor of 5-10 and outside the error margins. This deviation is too large to be compensated by other launch parameters. Therefore values of $\lambda_{z \text {, launch }}^{*}<1.5 \mathrm{~km}$ are not realistic. On the other hand for $\lambda_{z \text {,launch }}^{*}>6 \mathrm{~km}$ the GW-MF model values exceed CRISTA-1 GW-MF by over a factor of 4-5, suggesting that also values $\lambda_{z \text {,launch }}^{*}>6 \mathrm{~km}$ are not realistic.

The different choices of $s$ in Figs. 11a-c have almost no effect on this general behavior. Solely on average the model values for $s=0.5$ (Fig. 11a) are somewhat higher and the model values for $s=2$ (Fig. 11c) are somewhat lower than the results for $s=1$ (Fig. 11b). As a consequence, the contour line labeled " 1 " is slightly shifted towards lower values of $\lambda_{z \text {,launch }}^{*}$ for $s=0.5$ and towards higher values $\lambda_{z \text {, launch }}^{*}$ for $s=2$.

In Figs. 11d-f correlations between the horizontal distributions of Warner and McIntyre and CRISTA-1 GW-MF absolute values are shown for (d) $s=0.5$, (e) $s=1$, and (f) $s=2$. Similar as in Fig. 5 the correlation increases with altitude. Except for the lowest values $\lambda_{z \text {,launch }}^{*}<1 \mathrm{~km}$ the correlation is almost independent from $\lambda_{z \text {, launch. }}^{*}$.

In Figs. 12a-c the deviations of GW-MF values between model and instrument for the $464 \mathrm{mbar}$ launch level are given 

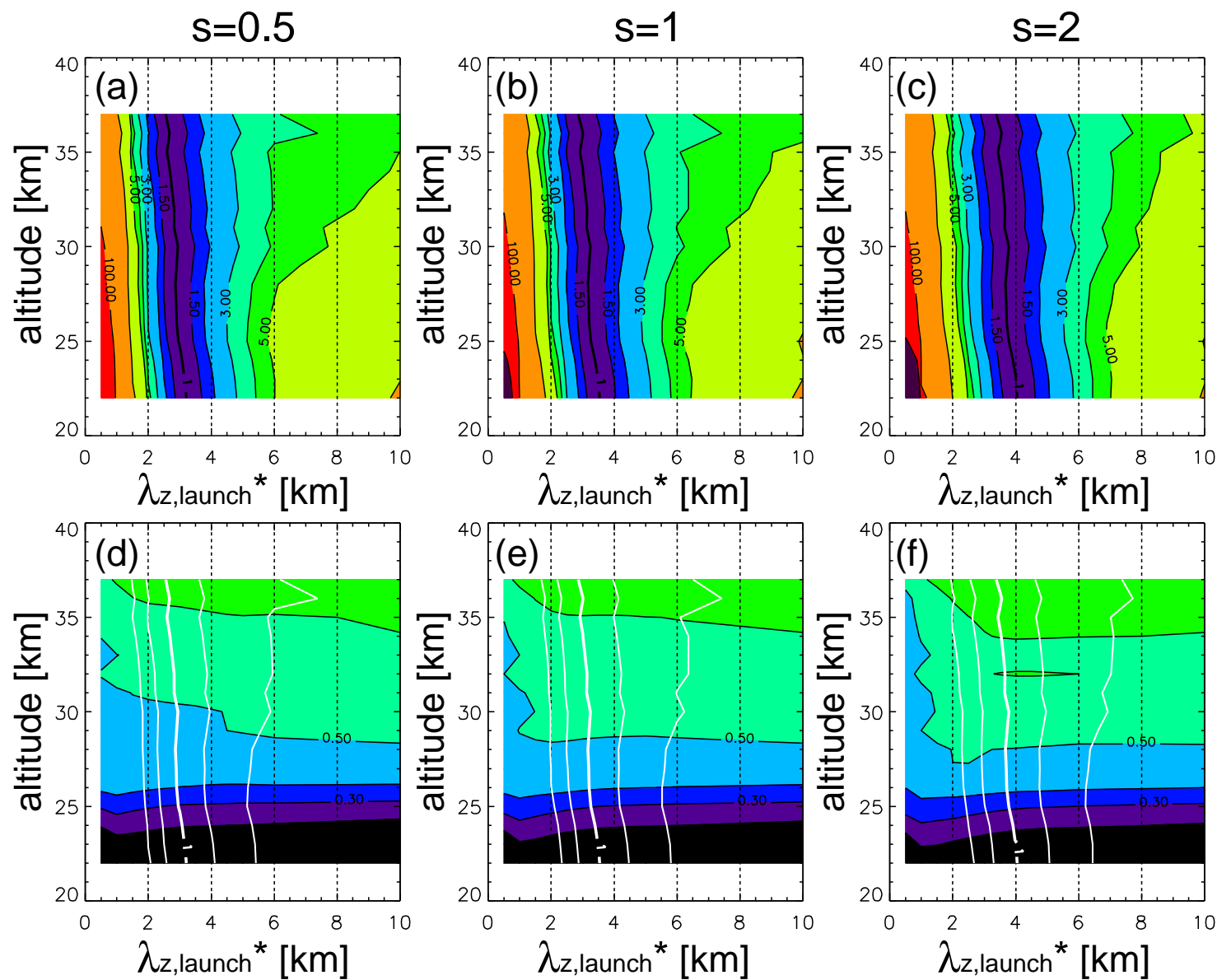

Fig. 11. Shown are for the Warner and McIntyre/CRISTA-1 comparison versus altitude and $\lambda_{z \text {, launch }}^{*}=2 \pi / m_{\text {launch }}^{*}$ :

(a-c) deviations of the Warner and McIntyre GW-MF absolute values (launch level 464 mbar) from CRISTA-1 as a reference, reciprocal taken from values $<1$ (at low $\lambda_{z \text {,launch }}^{*}$ ). Contour lines are $1,1.5,2,3,4,5,10,100$, and 1000 .

(d-f) correlations between the horizontal distributions of Warner and McIntyre and CRISTA-1 GW-MF absolute values. Contour lines are: $0.2,0.3,0.4,0.5,0.6,0.7,0.75,0.8$. Overplotted white contour lines are the 1,2 and 4 contour lines for the deviation of absolute values shown in $(\mathrm{a}-\mathrm{c})$.

for the CRISTA-2 case. Again, (a) shows the results for $s=0.5$, (b) for $s=1$, and (c) for $s=2$. The general behavior is similar as for the CRISTA-1 comparison. However, there is a tilt between CRISTA-2 and model GW-MF absolute values with altitude (about a factor of 2 from 25 to $45 \mathrm{~km}$ ). For the Warner and McIntyre/CRISTA-2 comparison even values of $\lambda_{z \text {,launch }}^{*}$ as high as $8-10 \mathrm{~km}$ would be possible without too serious deviations between the magnitudes of model and CRISTA-2 GW-MF values. Again, the choice of $s$ causes only minor shifts of the absolute values.

Figures $12 \mathrm{~d}-\mathrm{f}$ show the correlation coefficients for the model vs. CRISTA-2 comparison with model launch level 464 mbar. The behavior of the correlation coefficients is completely different from the results obtained for the CRISTA-1 comparison. For CRISTA-2 correlation is maximum for low values of $\lambda_{z \text {, launch }}^{*}$, minimum for $\lambda_{z \text {, launch }}^{*}$ in the range of about $4-8 \mathrm{~km}$, and there is again higher corre- lation (but less pronounced) for $\lambda_{z \text {, launch }}^{*}>8 \mathrm{~km}$. The exact location of the maxima and minima changes with the choice of $s$. Especially the choice of $s=2$ produces a broader range of maximum correlation at values $\lambda_{z \text {, launch }}^{*}$ in the range from about $1-4 \mathrm{~km}$.

\subsubsection{Variation of $\lambda_{z \text {, launch }}^{*}$ and $s$ : discussion of results}

Determining a best choice global set of launch parameters for the Warner and McIntyre scheme, i.e. suitable values of $\lambda_{z, \text { launch }}^{*} s$ and the launch level, means to weight the different results appropriately and to compromise between the two CRISTA flights. This will be aimed at in the following discussion. To decide whether a certain combination of $\lambda_{z, \text { launch }}^{*}$ and $s$ provides good agreement of CRISTA and model GWMF two criteria have to be fulfilled.

As a first criterion the deviation between CRISTA and 

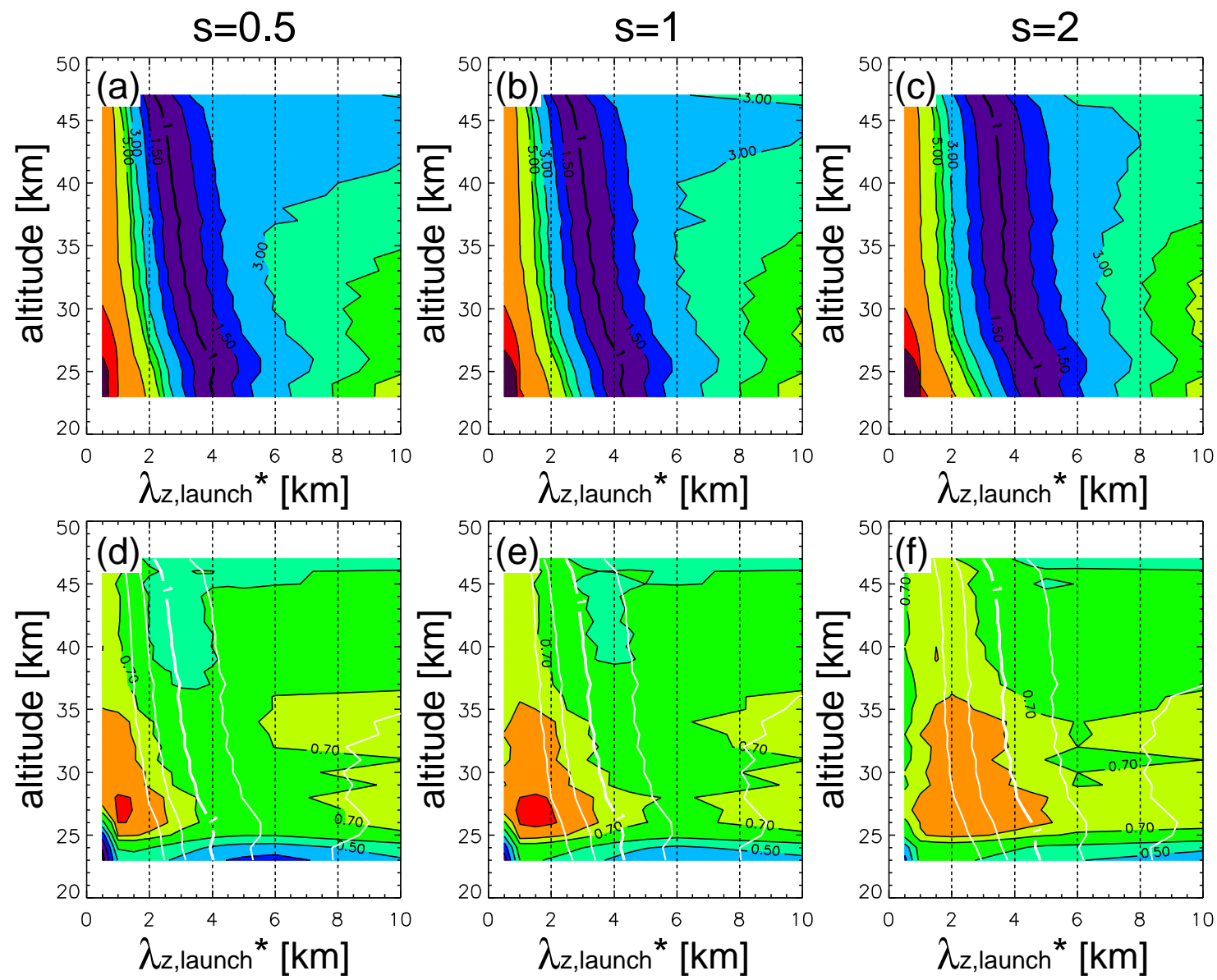

Fig. 12. Shown are for the Warner and McIntyre / CRISTA-2 comparison versus altitude and $\lambda_{z \text {, launch }}^{*}=2 \pi / m_{\text {launch }}^{*}$ : (a-c) deviations of the Warner and McIntyre GW-MF absolute values (launch level 464 mbar) from CRISTA-2 as a reference, reciprocal

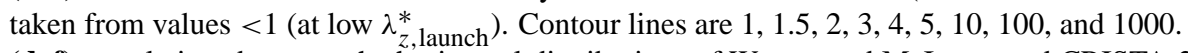

(d-f) correlations between the horizontal distributions of Warner and McIntyre and CRISTA-2 GW-MF absolute values. Contour lines are: $0.2,0.3,0.4,0.5,0.6,0.7,0.75,0.8$. Overplotted white contour lines are the 1,2 and 4 contour lines for the deviation of absolute values shown in $(\mathrm{a}-\mathrm{c})$.

Warner and McIntyre values of GW-MF can be used (absolute value criterion). The deviation between CRISTA and Warner and McIntyre results should not exceed a factor of about 4-5 given by the error ranges of the data. The distribution of the correlations shown in Figs. 11 and 12 can serve as a second criterion (correlation criterion): The ranges of $\lambda_{z \text {,launch }}^{*}$ with the highest correlation will be favored.

The general behavior of the GW-MF deviations, apart from some tilt with altitude between CRISTA and model GW-MF values and some shifts in the model value due to different choices of $s$, is as follows: The deviations are minimum for $\lambda_{z, \text { launch }}^{*}$ in the range of about $2.5-4 \mathrm{~km}$, model values are too low for about $\lambda_{z \text {,launch }}^{*}<2 \mathrm{~km}$ and too high for $\lambda_{z \text {,launch }}^{*}>6-10 \mathrm{~km}$. More exact values for the different choices of $s$ are summarized in Table 1 in the column for the absolute value criterion. This gives a first constraint to the possible range of $\lambda_{z, \text { launch }}^{*}$.
As can be seen from Figs. 11a-c for the CRISTA-1 case there are almost no further constraints from the correlation criterion.

There is much stronger variation of the correlation for CRISTA-2. Usually there is a region of high correlation at low values of $\lambda_{z, \text { launch }}^{*}$ ranging from low to high altitudes and also a region of higher correlation at values of $\lambda_{z \text {,launch }}^{*}>6 \mathrm{~km}$. The region at high $\lambda_{z \text {,launch }}^{*}$ shows high correlation only at altitudes $25-40 \mathrm{~km}$ and correlation is lower at lower and higher altitudes. Nevertheless, this region is listed in Table 1 for the sake of completeness.

Resulting $\lambda_{z \text {,launch }}^{*}$ ranges (see Table 1) have been determined by combining the limitations given by the absolute value and the correlation criterion. In addition, the resulting ranges have been limited to values of $\lambda_{z}^{*}$, launch $\leq 4 \mathrm{~km}$ because values of $\lambda_{z \text {, launch }}^{*}$ higher than about $4 \mathrm{~km}$ result in too high $\lambda_{z}^{*}$ in the lower stratosphere (see Sect. 4.2). 
Table 1. Possible $\lambda_{z \text {,launch }}^{*}$ ranges for the second lowest launch level (464 mbar, i.e. $\sim 5.4 \mathrm{~km}$ ).

\begin{tabular}{|c|c|c|c|c|}
\hline$s$ & $\begin{array}{c}\lambda_{z, \text { launch }}^{*} \text { range }[\mathrm{km}] \\
\text { (absolute value criterion) }\end{array}$ & $\begin{array}{l}\lambda_{z, \text { launch }}^{*} \text { range }[\mathrm{km}] \\
(\text { correlation criterion })\end{array}$ & $\begin{array}{l}\text { resulting } \lambda_{z, \text { launch }}^{*} \text { range }[\mathrm{km}] \\
\text { both criteria combined, cutoff } 4 \mathrm{~km}\end{array}$ & $\begin{array}{c}\text { optimum } \\
\lambda_{z, \text { launch }}^{*}[\mathrm{~km}]\end{array}$ \\
\hline \multicolumn{5}{|c|}{ CRISTA-1 } \\
\hline 0.5 & $2.0-6.0$ & $1.0-10.0$ & $2.0-4.0$ & $2.5-4.0$ \\
\hline 1 & $2.0-6.5$ & $1.0-10.0$ & $2.0-4.0$ & $2.5-4.0$ \\
\hline 2 & $2.5-7.0$ & $1.5-10.0$ & $2.5-4.0$ & $3.0-4.0$ \\
\hline \multicolumn{5}{|c|}{ CRISTA-2 } \\
\hline 0.5 & $2.5-10.0$ & $1.0-2.0$ and $6.0-10.0$ & - & - \\
\hline 1 & $2.5-10.0$ & $1.0-3.0$ and $7.0-10.0$ & $2.5-3.0$ & 2.5 \\
\hline 2 & $2.5-10.0$ & $0.5-10.0$ & $2.5-4.0$ & $2.5-3.0$ \\
\hline
\end{tabular}

From Table 1 we can see that for CRISTA- 2 and $s=0.5$ the resulting range is empty. In the regions of high correlation the deviations from the CRISTA-2 GW-MF values are too large. This indicates that a common global value of $s=0.5$ would not be a good choice. Combining the resulting ranges of CRISTA- 1 and CRISTA- 2 for $s=1$ and $s=2$ gives us possible $\lambda_{z \text {,launch }}^{*}$ ranges of about $2.5-3$ and $2.5-4 \mathrm{~km}$, respectively. However, for CRISTA-2 and $s=1$ as a compromise we have to accept somewhat reduced correlation at altitudes above $35 \mathrm{~km}$. This situation is improved for $s=2$.

We have also determined optimum ranges of $\lambda_{z \text {, launch }}^{*}$ (see Table 1) by considering only the part of the resulting ranges with deviations less than a factor of about 2 for CRISTA1. For CRISTA-2 it is more important to choose $\lambda_{z, \text { launch }}^{*}$ in a way to obtain correlation as high as possible from low to high altitudes.

Therefore we choose as optimum values for CRISTA2 the lower limit of the resulting $\lambda_{z, \text { launch }}^{*}$ ranges. Combining the optimum values from CRISTA- 1 and CRISTA2 gives us optimum values of $\lambda_{z \text {, launch }}^{*}=2.5 \mathrm{~km}$ for $s=1$ and $\lambda_{z, \text { launch }}^{*}=3.0 \mathrm{~km}$ for $s=2$.

As can be summarized, a common global value of $\lambda_{z \text {, launch }}^{*}$ lower than about $2.0-2.5 \mathrm{~km}$ is unlikely because model GWMF is too low compared to CRISTA GW-MF. On the other hand, to preserve the high correlation for the CRISTA-2 case

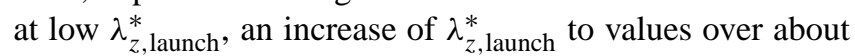
$3 \mathrm{~km}$ does not seem to be justified. This gives us a quite narrow range of $\lambda_{z, \text { launch }}^{*}$ which is compatible with both CRISTA missions for $s=1$ and $s=2$. Since $s=2$ gives the largest resulting range of $\lambda_{z \text {, launch }}^{*}$ this value might be better suited than $s=1$. For $s=0.5$ no resulting $\lambda_{z}^{*}$, launch range can be found for CRISTA-2. This means values of $s$ and $\lambda_{z \text {, launch }}^{*}$ somewhat higher than the standard values $s=1$ and $\lambda_{z \text {, launch }}^{*}=2 \mathrm{~km}$ are the best choice.

Optimum values of $\lambda_{z \text {, launch }}^{*}=2.5-3.0 \mathrm{~km}$ still produce a notable low-bias (about a factor of 2-3) of the model GW-
MF compared to CRISTA GW-MF. This low-bias could be reduced, for example, by increasing the model parameter $\beta$ (see Sect. 1) by a factor of 2. However, it should also be kept in mind that CRISTA GW-MF could be somewhat highbiased due to additional GW sources (e.g., mountain waves) not considered in the parameterization scheme and that deviations of a factor of 2-3 are inside the error limits.

\section{Influence of $\lambda_{z \text {,launch }}^{*}$ and $s$ on the vertical distribution of GW drag}

One of the main purposes of a GW parameterization scheme is to provide realistic values of GW drag so that winds calculated in GCMs are more reliable. In this section we investigate whether the ranges of $\lambda_{z, \text { launch }}^{*}$ and $s$ derived in the previous section are compatible with measurements and theoretical considerations. To check whether the different choices of launch parameters discussed above give reasonable results the zonal mean zonal GW drag has been calculated for some selected cases. As atmospheric background the same composite wind and temperature field was used as in (Preusse et al., 2006): From 0-28 km altitude European Centre for Medium-Range Weather Forecasts (ECMWF) reanalyses were used (Coy and Swinbank, 1997). For altitudes 20 $85 \mathrm{~km}$ CRISTA-2 temperatures and geostrophic wind derived from CRISTA-2 data were used (Oberheide et al., 2002). Above $70 \mathrm{~km}$ COSPAR International Reference Atmosphere (CIRA) climatological data were used (Chandra et al., 1990). At the overlapping altitudes smooth transitions were generated by applying weighted means.

The results obtained from the Warner and McIntyre scheme are shown in Fig. 13.

The peak values of GW drag are about the same in all cases shown, however, there are significant differences in the vertical distributions of GW drag. 

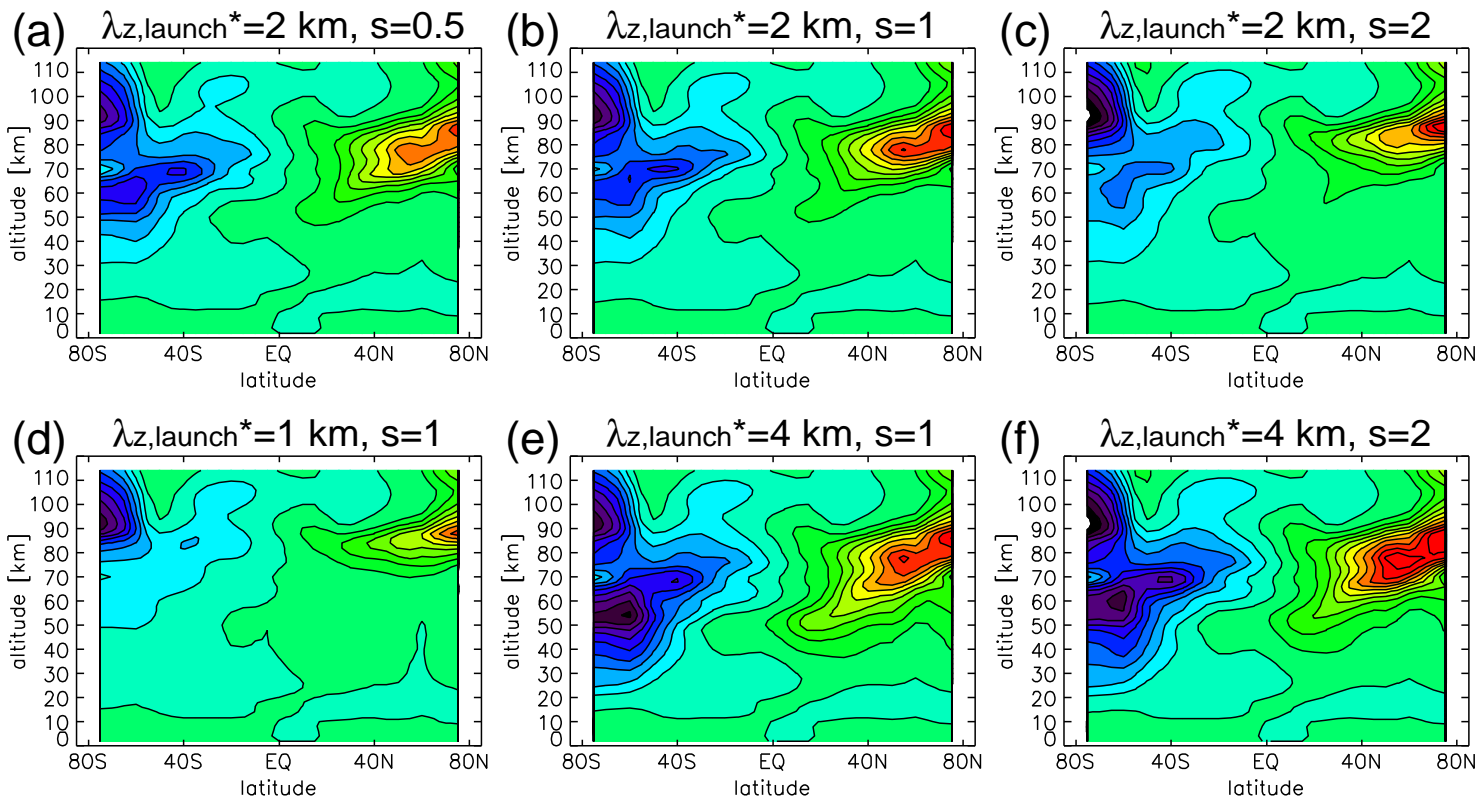

zonal mean zonal GW drag [m/s/day] \begin{tabular}{|lllllllll}
\hline & & & & & & & \\
\hline-60 & -40 & -20 & 0 & 20 & 40 & 60
\end{tabular}

Fig. 13. Zonal mean zonal GW drag in $\mathrm{m} / \mathrm{s}$ /day calculated with the Warner and McIntyre GW scheme for the CRISTA-2 case (August 1997) with launch level 464 mbar. Shown are results for the launch parameter combinations: (a) $\lambda_{z \text {, launch }}^{*}=2 \mathrm{~km}$ and $s=0.5$, (b) $\lambda_{z, \text { launch }}^{*}=2 \mathrm{~km}$ and $s=1$, (c) $\lambda_{z, \text { launch }}^{*}=2 \mathrm{~km}$ and $s=2$, (d) $\lambda_{z, \text { launch }}^{*}=1 \mathrm{~km}$ and $s=1$, (e) $\lambda_{z, \text { launch }}^{*}=4 \mathrm{~km}$ and $s=1$, (f) $\lambda_{z, \text { launch }}^{*}=4 \mathrm{~km}$ and $s=2$.

Figures 13a-c show the influence of different values $s$ for fixed $\lambda_{z, \text { launch }}^{*}=2 \mathrm{~km}$. For $s=0.5$ (Fig. 13a) GW-MF is higher in the unsaturated part of the launch spectrum than for the standard case with $s=1$ (Fig. 13b). Therefore for $s=0.5$ a larger part of the GW spectrum is saturated already at lower altitudes, leading to somewhat higher GW drag already at lower altitudes than in the standard case (see Figs. 13a and b). Accordingly, for $s=2$ (Fig. 13c) a shift of high GW drag towards higher altitudes would be expected because GW-MF in the unsaturated part of the launch spectrum is reduced and GW breaking postponed towards higher altitudes. Comparing Figs. $13 \mathrm{~b}$ and $\mathrm{c}$ this is observed, indeed.

Figures $13 \mathrm{~d}-\mathrm{f}$ show the influence of different $\lambda_{z, \text { launch }}^{*}$ on the GW drag vertical distribution. The results of Fig. 13d were obtained with $\lambda_{z \text {, launch }}^{*}=1 \mathrm{~km}$ and $s=1$. This means a larger part of the launch spectrum is unsaturated. And, as expected, in Fig. 13d the regions of GW breaking and high GW-MF are shifted to higher altitudes than in the standard case with $\lambda_{z \text {, launch }}^{*}=2 \mathrm{~km}$ and $s=1$ (Fig. 13b). For example, in Fig. $13 \mathrm{~d}$ at the southernmost latitudes there is a peak of GW drag at altitudes above $90 \mathrm{~km}$ and only moderate values of GW drag below. This distribution of GW drag does not seem to be realistic because there are indications for GW breaking already at lower altitudes of about 50-60 km at the top of the southern polar jet (Preusse et al., 2006). Therefore higher values of GW drag are expected already in the altitude region
$50-60 \mathrm{~km}$. This confirms that the choice of $\lambda_{z \text {,launch }}^{*}=1 \mathrm{~km}$ is too low.

In Fig. 13e the launch parameters were $\lambda_{z \text {,launch }}^{*}=4 \mathrm{~km}$ and $s=1$. Choosing a higher value of $\lambda_{z \text {, launch }}^{*}$ implicates a larger saturated part of the launch spectrum. Correspondingly in Fig. 13e already at altitudes as low as $30 \mathrm{~km}$ relatively high GW drag values of about $10-15 \mathrm{~m} / \mathrm{s} /$ day can be found in the region of the southern polar jet. Increasing $s$ to a value of 2 (Fig. 13f) cannot reduce this effect significantly.

Using a value of $\lambda_{z, \text { launch }}^{*}=10 \mathrm{~km}$ (not shown) leads to GW drag values of up to $50 \mathrm{~m} / \mathrm{s} /$ day already at altitudes of $25 \mathrm{~km}$. From theoretical considerations maximum values of about $2 \mathrm{~m} / \mathrm{s} /$ day at these altitudes would be expected (e.g., Alexander and Rosenlof, 1996). Measurements can exceed this value by more than a factor of two (e.g., Sato, 1994). Nevertheless, the very high values of GW drag at low altitudes obtained for $\lambda_{z \text {, launch }}^{*}=10 \mathrm{~km}$ seem to be unrealistic. An increase of $\lambda_{z \text {, launch }}^{*}$ over a value of about $4 \mathrm{~km}$ therefore does not seem to make sense. This gives us another consistency check for the reasonable ranges of $\lambda_{z \text {, launch }}^{*}$ deduced from Table 1 .

In the Warner and McIntyre scheme the parameter $\beta$ (see also Sect. 1) is proportional to the values of GW-MF as well as to the values of GW drag. Therefore scaling of GWMF and GW drag with $\beta$ as suggested in Sect. 4.3.2 can be used to reduce the low-bias of model GW-MF without 


\section{CRISTA-2}
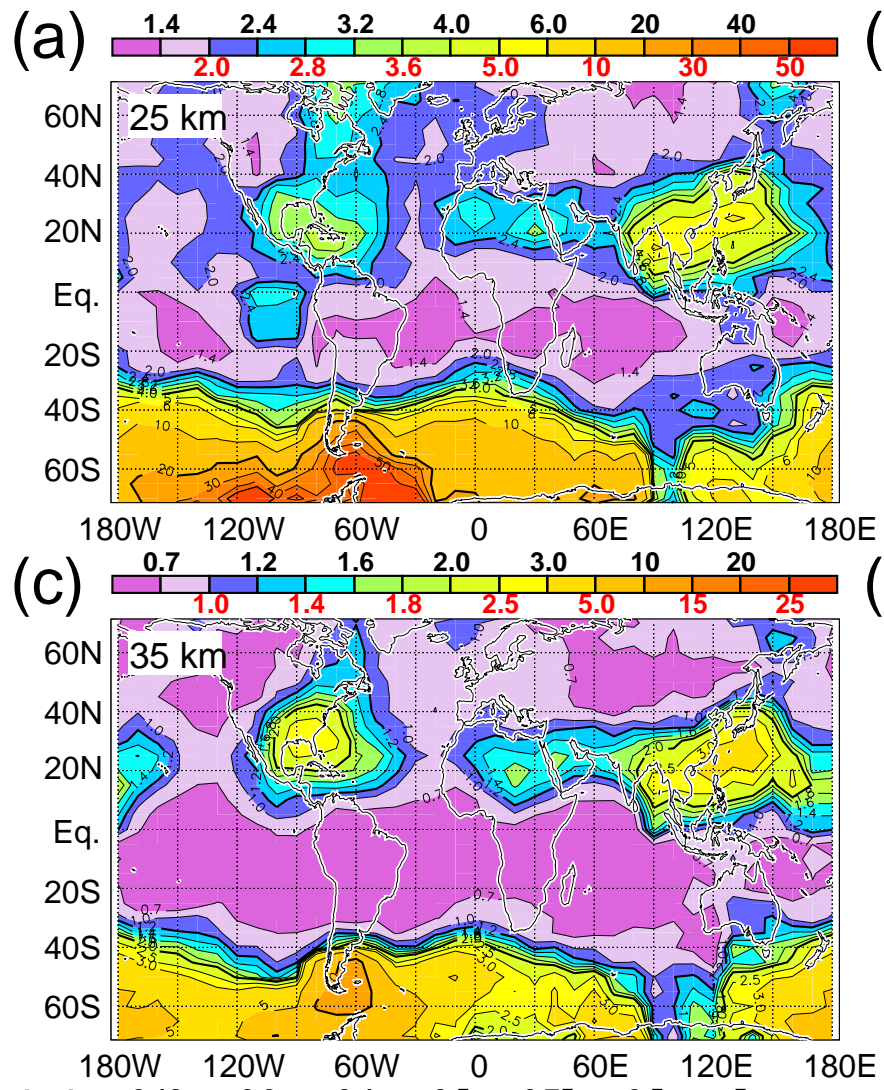
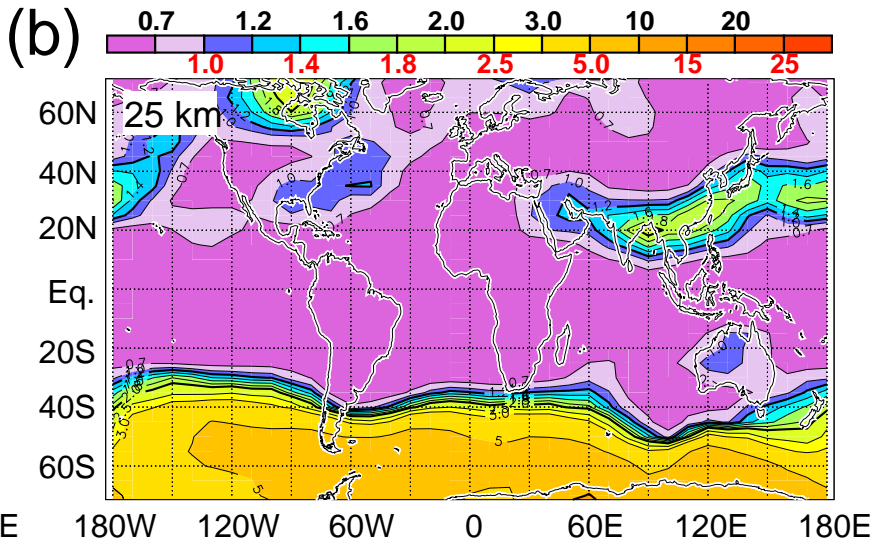
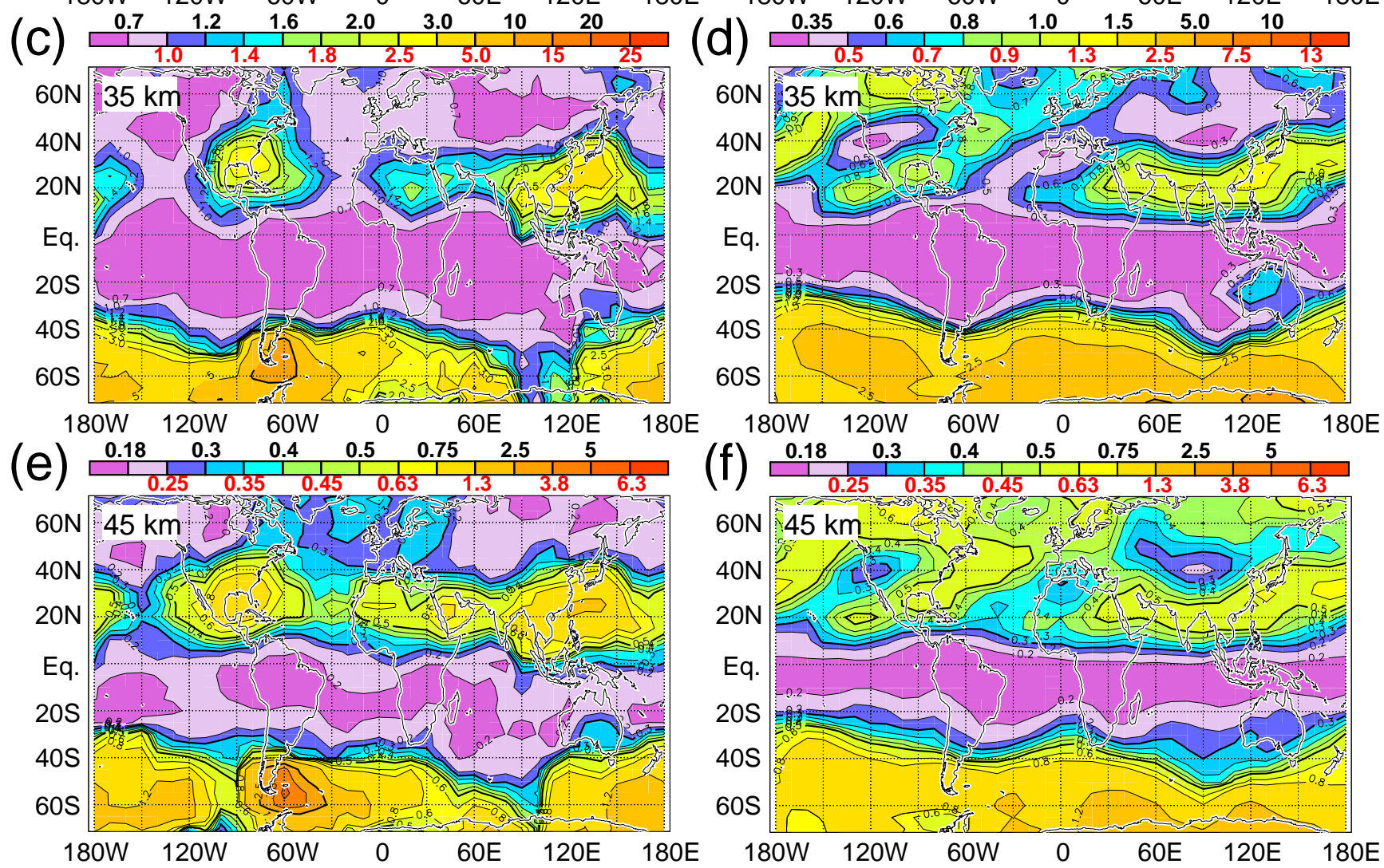

Fig. 14. Shown are horizontal distributions of GW-MF absolute values in mPa (see color bars and contour labels) for CRISTA-2 (August 1997) at altitudes of $25 \mathrm{~km}$ (a), $35 \mathrm{~km}$ (c), and $45 \mathrm{~km}(\mathbf{e})$. Also shown are horizontal distributions of GW-MF absolute values in mPa calculated with the Warner and McIntyre GW parameterization scheme at the same altitudes (25 km (b), $35 \mathrm{~km}(\mathbf{d})$, and $45 \mathrm{~km}(\mathbf{f}))$ for a fixed model GW launch level at 464 mbar (about $5.4 \mathrm{~km}$ ) and the spectral parameters $s=2$ and $\lambda_{z}^{*}$. launch $=3 \mathrm{~km}$. Instrumental visibility filtering has been applied to the model values. Please note that due to differences in the GW-MF absolute values contour lines and color codes are different at different altitudes and also different for CRISTA and model results.

Figure 3a was reproduced from Fig. 3d in: Ern, M., Preusse, P., Alexander, M. J., and Warner, C. D., Absolute values of gravity wave momentum flux derived from satellite data, J. Geophys. Res., 109, D20103, doi:10.1029/2004JD004752, 2004. Copyright [2004] American Geophysical Union. Reproduced by permission of American Geophysical Union. 

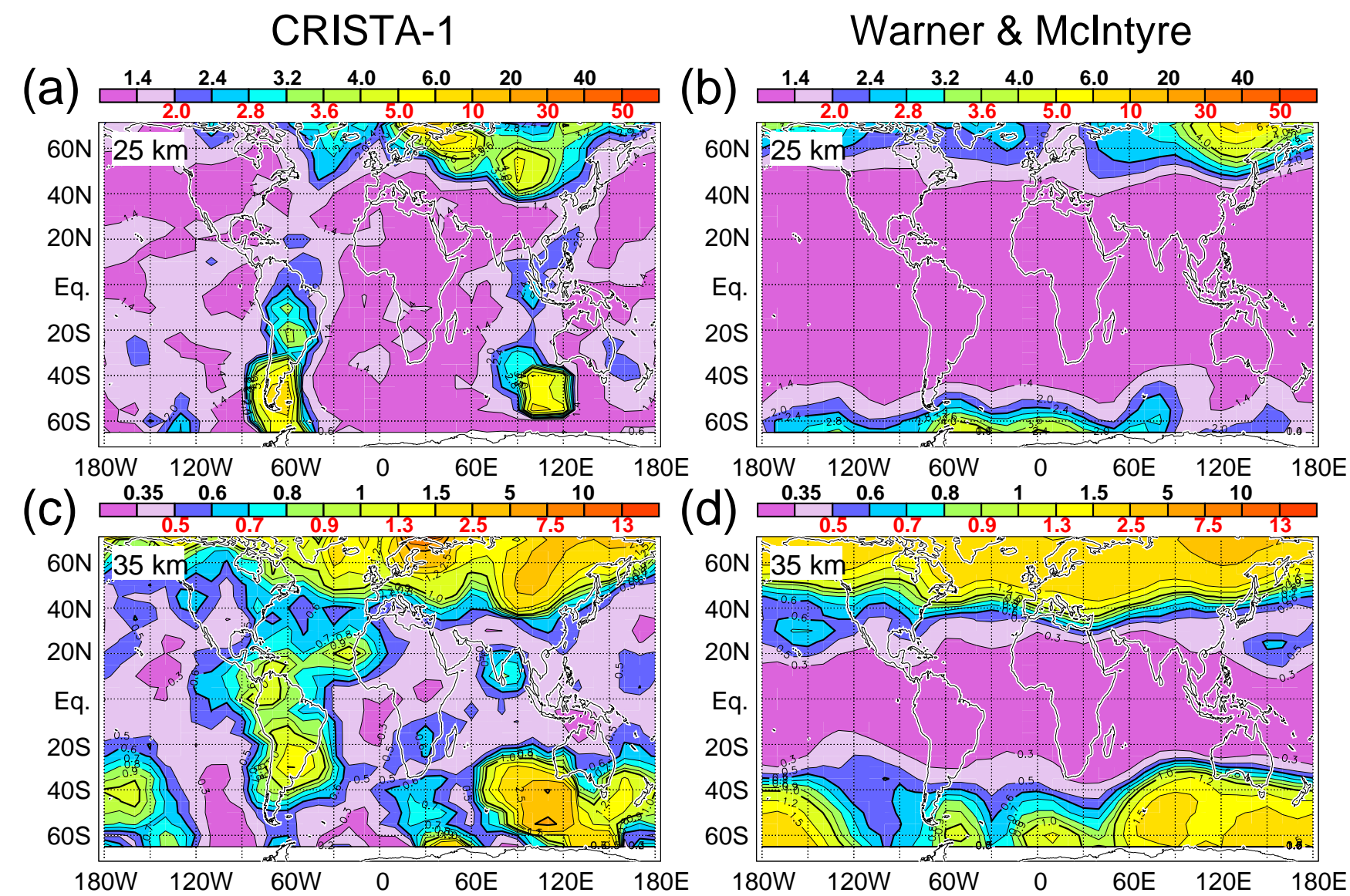

Fig. 15. Horizontal distributions of GW-MF absolute values in $\mathrm{mPa}$ (see color bars and contour labels) for CRISTA-1 (November 1994) at altitudes of $25 \mathrm{~km}$ (a) and $35 \mathrm{~km}$ (c). Also shown are horizontal distributions of GW-MF absolute values in mPa calculated with the Warner and McIntyre GW parameterization scheme at the same altitudes $(25 \mathrm{~km}(\mathbf{b}), 35 \mathrm{~km}(\mathbf{d}))$ for a fixed model GW launch level at $464 \mathrm{mbar}$ about $5.4 \mathrm{~km}$ ) and the spectral parameters $s=2$ and $\lambda_{z \text { launch }}^{*}=3 \mathrm{~km}$. Instrumental visibility filtering has been applied to the model values. Please note that due to differences in the GW-MF absolute values contour lines and color codes are different at different altitudes and also different for CRISTA and model results.

changing the relative distributions of GW-MF and GW drag. This means the correlation between modeled GW-MF and CRISTA GW-MF as reference is left unchanged and also the ranges of launch parameters determined from the correlation criterion.

Indeed, increasing of the model parameter $\beta$ (see Sect. 1) to reduce the low-bias of model GW-MF as suggested in Sect. 4.3.2 makes sense because for the CRISTA-2 case peak values of acceleration calculated with the Warner and McIntyre scheme in the upper mesosphere are about $50 \mathrm{~m} / \mathrm{s} /$ day for the standard launch parameters (see Fig. 13b) and only a little higher for the optimum launch parameters shown in Table 1. On the other hand monthly mean values of GWMF derived from radar observations can be as high as 100$200 \mathrm{~m} / \mathrm{s}$ /day (Hocking, 2005). This means higher values of $\beta$ than the standard value of $\sim 0.1$ are not in contradiction with observations. In fact, a value of $\beta=0.2$ would result in peak values comparable to those reported by Hocking (2005).

Another important point can be seen from Fig. 13: There are high values of GW drag not only in the winter hemisphere at the top of the southern polar jet, but also in the northern hemisphere where only little GW-MF was observed by CRISTA (see above) and also only low temperature variances are observed at higher altitudes (Preusse et al., 2006). This means that GW-MF and GW drag are too high in the model results. Again, this is a clear indication that the assumption of a global launch distribution for GW parameterization schemes is too simple and global measurements of GW-MF especially in the mesosphere and the mesopause region over a full annual cycle are in need to give further constraints to the GW-MF launch distribution. 

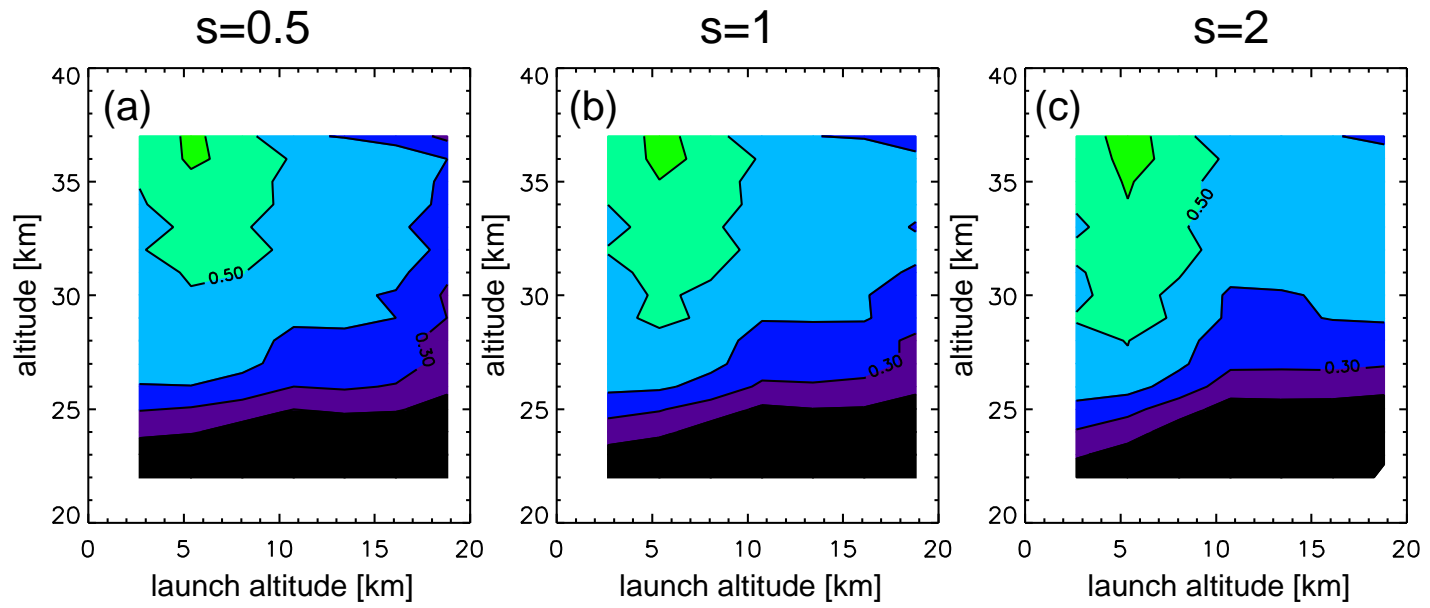

Fig. 16. Shown are for the Warner and McIntyre/CRISTA-1 comparison of horizontal GW-MF distributions correlation coefficients versus altitude and model launch altitude for the choice of $\lambda_{z \text {, launch }}^{*}=3 \mathrm{~km}$ and $s=0.5$ (a), $s=1$ (b), and $s=2.0$ (c). Contour lines are: 0.2, 0.3, 0.4, 0.5, 0.6 .
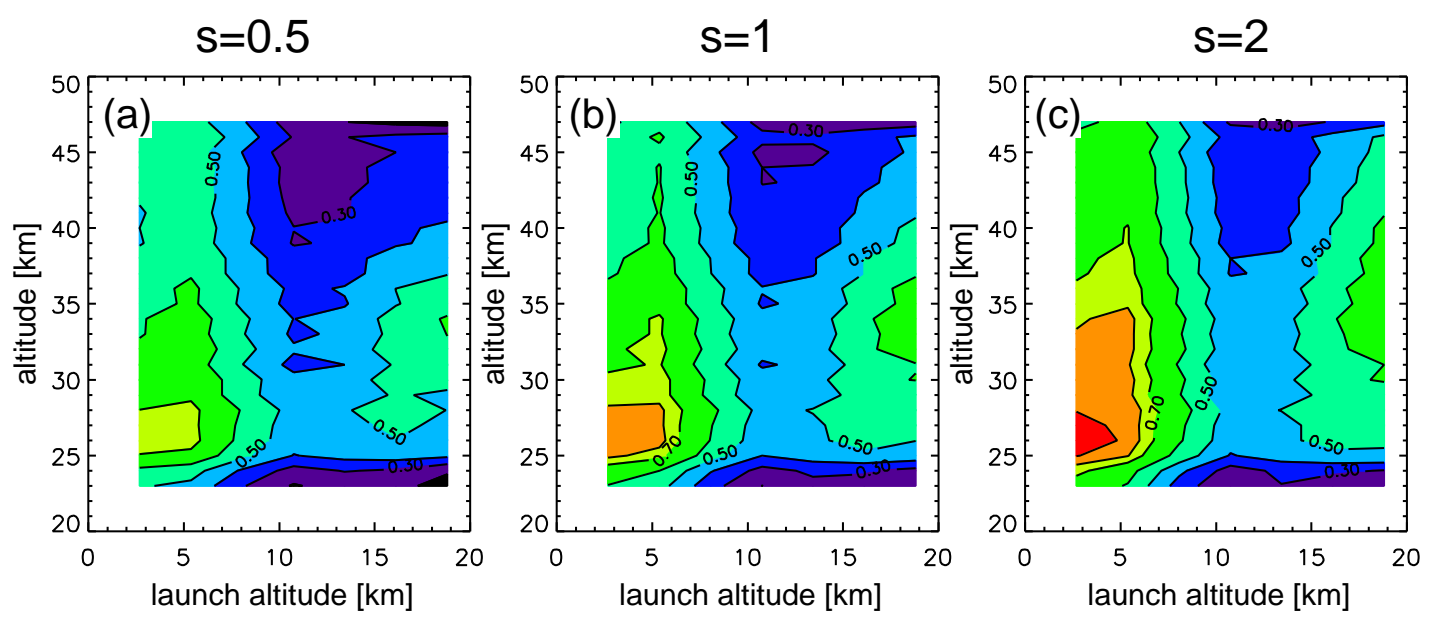

Fig. 17. Same as Fig. 16, but for CRISTA-2. Contour lines are: $0.2,0.3,0.4,0.5,0.6,0.7,0.75,0.8$.

\section{Summary and conclusions}

In this paper absolute values of GW-MF derived from the CRISTA-1 (November 1994) and CRISTA-2 (August 1997) satellite missions have been compared to GW-MF absolute values calculated with the Warner and McIntyre GW parameterization scheme for these two periods. Horizontal structures of GW-MF can be reproduced already by using the standard set of Warner and McIntyre launch parameters $\left(\lambda_{z, \text { launch }}^{*}=2 \mathrm{~km}\right.$ and $\left.s=1\right)$ if a low launch level is used. For this standard set of launch parameters the model values of GW-MF are considerably lower, however, this set of parameters is already some kind of compromise, considering the large error range of about a factor of $4-5$ for the GW-MF absolute values.

The best correlation between CRISTA and Warner and
McIntyre horizontal distributions is achieved for the second lowest model launch level $464 \mathrm{mbar}$ (i.e. about $5.4 \mathrm{~km}$ ). This maximum correlation is a persistent feature for both CRISTA flights and almost all choices of spectral launch parameters $\lambda_{z \text {,launch }}^{*}$ and $s$.

Possible ranges of $\lambda_{z, \text { launch }}^{*}$ have been determined by optimizing the agreement of GW-MF absolute values as well as the correlation between CRISTA and model distributions of GW-MF and by considering the vertical distribution of $\lambda_{z}^{*}$. The resulting range for $\lambda_{z, \text { launch }}^{*}$ is about $2-4 \mathrm{~km}$, depending on $s$. The value $s=2$ gives better overlap between the ranges obtained for the absolute value criterion on the one hand and the correlation criterion on the other hand. Using the vertical distribution of GW drag as cross-check confirms the derived $\lambda_{z \text {,launch }}^{*}$ range of about $2-4 \mathrm{~km}$. 

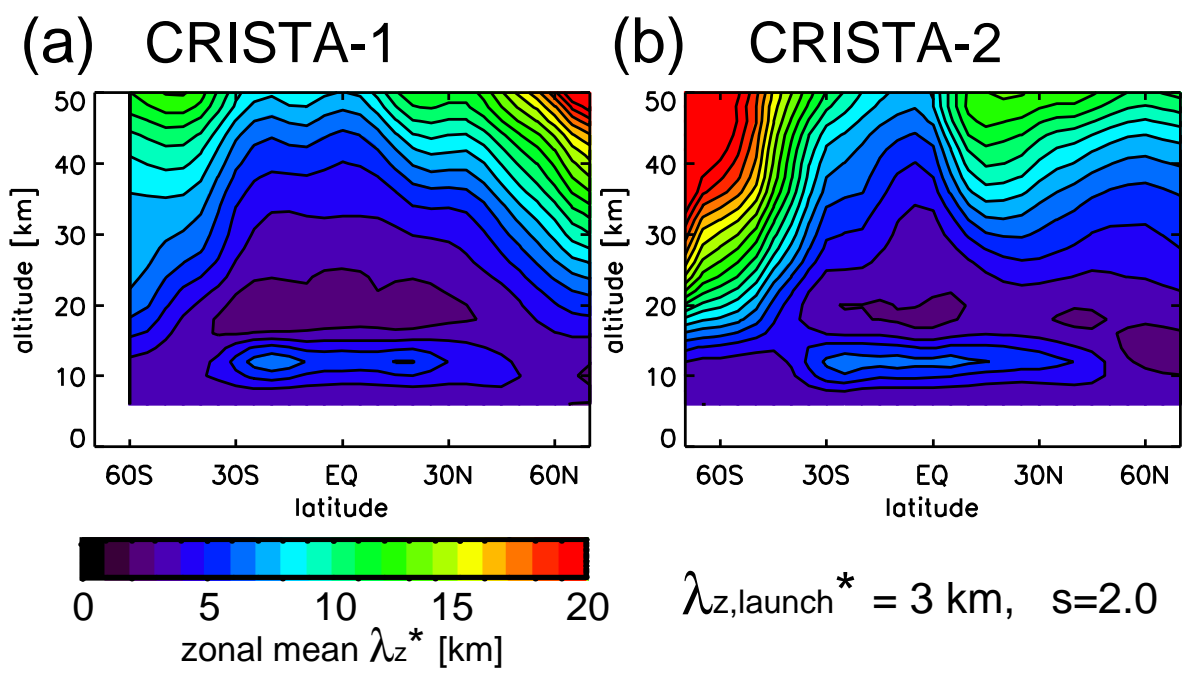

Fig. 18. Zonal mean cross section of $\lambda_{z}^{*}$ obtained from the Warner and McIntyre scheme (launch level $464 \mathrm{mbar}, \lambda_{z, \text { launch }}^{*}=3 \mathrm{~km}$, and $s=2$ ) for the direction of maximum GW-MF during CRISTA-1 (a) and CRISTA-2 (b). No observational filter was applied.

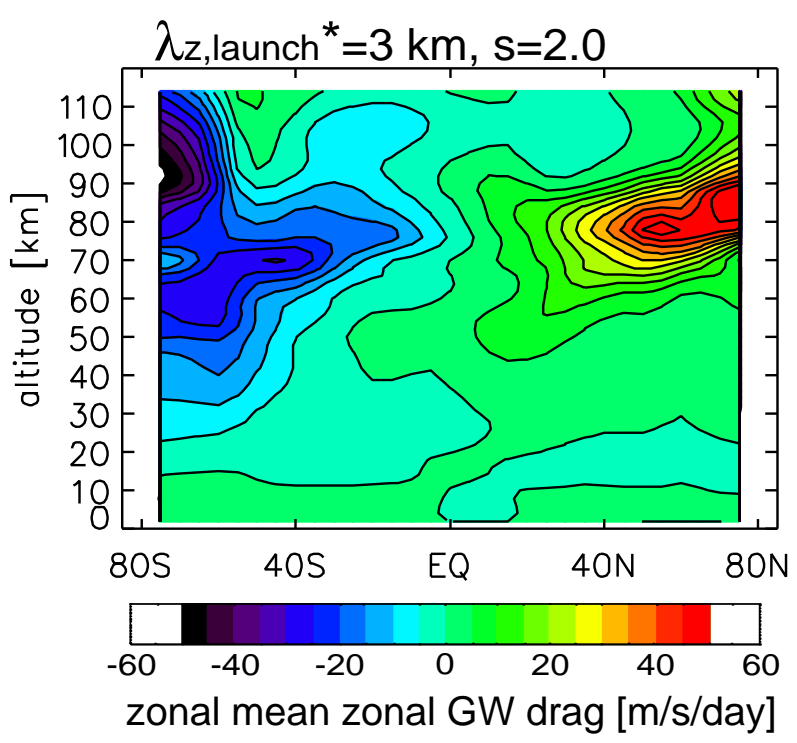

Fig. 19. Zonal mean zonal GW drag in $\mathrm{m} / \mathrm{s} /$ day calculated with the Warner and McIntyre GW scheme for the CRISTA-2 case (August 1997) using the optimum launch parameters: launch level 464 mbar, $\lambda_{z, \text { launch }}^{*}=3 \mathrm{~km}$ and $s=2$.

This means there are some indications that $\lambda_{z \text {,launch }}^{*}$ should be somewhat larger than the standard value of $2 \mathrm{~km}$, but not very much larger (maybe $\lambda_{z \text {, launch }}^{*}=2.5-3.0 \mathrm{~km}$ ). In addition, the parameter $s$ should be increased to $s=2$, reducing the spectral power in the unsaturated part of the GW vertical wavenumber spectrum. The remaining low-bias of the model GW-MF with respect to the CRISTA estimates could be reduced by increasing the model input parameter $\beta$ by a factor of about 2 (this would also increase the GW-MF values as well as the GW drag values by a factor of 2) without causing incompatibilities with radar observations of GW drag.

The choice of such a global set of model launch parameters should be made with some caution for some reasons. First, the error ranges of GW-MF are relatively large, even though part of the error will be a systematic error and relative variations of GW-MF in the horizontal distributions are highly significant. Of course, this large error range, as well as the fact that CRISTA is only a very limited data set of two weeks of measurements in a limited altitude interval, will put some uncertainty on the determined ranges of model parameters. Second, the choice of a global set of launch parameters itself is a problem. Already from the CRISTA versus Warner and McIntyre model comparison there are some indications for localized GW sources which cannot be reproduced by the model. In addition, there are high values of model GW drag in northern latitudes and at the same time only little GW activity at high northern latitudes during CRISTA-2. This indicates that there should be some annual cycle in the GW sources at middle and high latitudes which is not incorporated in the model.

Therefore we conclude that to overcome the limitation of GW parameterization schemes to a fixed set of launch parameters detailed global measurements of the GW source distribution over a full annual cycle are highly desirable. 


\section{Appendix A}

\section{Separation of GW fluctuations by horizontal Kalman filtering}

A problem which is common to nearly all experimental work on GWs is that the atmospheric fluctuations caused by GWs need to be discerned from other kinds of variations by a scale separation approach. Evidently, several problems arise: First, there might exist other phenomena in the atmosphere inside the scales considered as GWs, second, other phenomena could leak into the scales considered as GWs, third, GWs can have scales outside the limits of the scale separation approach, and, fourth, the applied detrending algorithm can redistribute GW energy in space or wavelength.

Apparently, the third is the smallest problem, because the detrending can be specified and the results therefore properly characterized. However, a too limited wavelength range can make interpretations very difficult (e.g., Alexander, 1998; Preusse et al., 2006).

Validation, whether the scale separation approach is working properly, can be performed in three ways: First, the GW dispersion or polarization relations can be used to actually prove that an observed pattern is a GW. This requires, however, to measure either the horizontal and vertical wavelength as well as the frequency of the wave to test the dispersion relation or the wind and temperature amplitudes as well as two of the above mentioned quantities to test the polarization relation. Such tests have been performed in case studies, e.g., for radio sondes (polarization relation) and for CRISTA data investigating a large scale wave observed above supertyphoon Winnie (Preusse et al., 2001). If it can be assumed that the observed waves are mountain waves, the dispersion relation can be tested with the vertical wavelength alone (Eckermann and Preusse, 1999; Preusse et al., 2002). Second, one can try to understand in case studies the nature of the waves, e.g., one can perform regional or global modeling and show that salient features of the waves or global distributions match (e.g., Eckermann and Preusse, 1999; Preusse et al., 2002, or, to quote different measurement techniques, Dewan et al., 1998; Jiang et al., 2004a). One also can sometimes compare to proxies of GW sources and find reasonable agreement in the distribution patterns as well as wave characteristics matching with the sources (e.g., Preusse et al., 2001; Jiang et al., 2004b). This can raise the confidence that in general the patterns retrieved by the scale separation approach are best explained in terms of GWs though it is not a strict proof. Third, one can try to think of different processes, such as balanced motions, and estimate their influence on the estimated GW distributions. This approach has two disadvantages: First, one investigates only what comes to ones mind and the investigation can therefore never be complete, and second, it can be performed only in case studies, too.

How can we apply this discussion to the Kalman filter? There is a large number of case studies (the quoted refer- ences are only a few examples) which fall in the first and second category. There are, in addition, some findings indicating that the scale separation by the Kalman filter works. We have run the Kalman filter up to wave number 16. However, for temperatures most of the spectral power is contained in the first three wave numbers and the amplitudes at wave numbers higher than six are small compared to average GW amplitudes at corresponding latitudes. On the other hand high wave numbers of the Kalman filter are required to map balanced-motion signatures in trace gases, such as streamers and filaments (cf. Offermann et al., 1999). We have also compared GW patterns with the location of streamers and filaments in CRISTA data. In general there are no enhanced GWs connected with such patterns. There is a heuristic argument why balanced motions do not contribute largely to the observed temperature fluctuations. Any wave-like temperature structure in the atmosphere experiences damping due to dissipation as well as radiative transfer. The estimated typical damping time scales for waves depend on horizontal and vertical wavelengths and are of the order of one day for structures of the size of streamers and filaments (Fels, 1982, 1984; Marks and Eckermann, 1995). Typical lifetimes of structures in tracers are of the order of one week. The abundance of streamers and filaments in tracers is therefore likely not reflected in corresponding temperature signals.

Based on these three arguments, i.e., tests of the dispersion relation, explanation of wave properties and global distributions, and observed power decrease at higher wavenumbers, we are therefore confident that the Kalman filter truly isolates GWs from other kind of signatures in the stratosphere and mesosphere. Problems could be posed by fast propagating waves, though of global scale, as, e.g., the two-day wave and ultra-fast Kelvin waves. Here, analyzing momentum flux instead of temperature fluctuations or wave potential energy has the additional advantage of focusing on the shorter horizontal wavelengths and thereby strengthening the scale separation approach. Compared to the uncertainties introduced by instrumental noise, distortions from the radiative transfer and, in particular, the undersampling of the horizontal wave structure, the uncertainties due to the scale separation approach can be considered as small.

\section{Appendix B}

\section{Figures for optimum launch parameters}

In this appendix some of the figures are reproduced for comparison with the optimum choice of launch parameters (launch level $464 \mathrm{mbar}, \lambda_{z, \text { launch }}^{*}=3 \mathrm{~km}$ and $s=2$ ).

Figures 14 and 15 show the horizontal maps calculated with the Warner and McIntyre scheme for the CRISTA-1 and the CRISTA-2 period with the CRISTA GW-MF as reference, respectively, at different altitudes (see also Figs. 3 and 4). The following optimum set of launch parameters was 
used for the Warner and McIntyre results: a fixed model GW launch level at $464 \mathrm{mbar}$ (about $5.4 \mathrm{~km}$ ) and the spectral parameters $s=2$ and $\lambda_{z, \text { launch }}^{*}=3 \mathrm{~km}$.

Figures 16 and 17 show the correlation coefficient vs. altitude and launch altitude for CRISTA-1 (Figs. 16) and CRISTA-2 (Fig. 17) for $\lambda_{z, \text { launch }}^{*}=3 \mathrm{~km}$ and different choices of $s$. Figures $16 \mathrm{c}$ and $17 \mathrm{c}(s=2.0)$ are for the optimum set of launch parameters mentioned above. For comparison see also Figs. 5 and 6.

Figure 18 shows the latitude altitude cross section of $\lambda_{z}^{*}$ for the optimum set of launch parameters (launch level 464 mbar, $\lambda_{z, \text { launch }}^{*}=3 \mathrm{~km}$, and $s=2$ ) for CRISTA-1 (Fig. 18a) and CRISTA-2 (Fig. 18b). For comparison see also Figs. 9 and 10.

Figure 19 shows the zonal mean zonal GW drag in $\mathrm{m} / \mathrm{s} /$ day vs. latitude and altitude for the optimum set of launch parameters (see above) for the CRISTA-2 period (August 1997). For comparison see also Fig. 13

Acknowledgements. This work was funded through Deutsche Forschungsgemeinschaft (DFG) project GW-CODE (ER 474/1-1), DFG priority program CAWSES SPP 1176.

Edited by: K. Hamilton

\section{References}

Alexander, M. J. and Rosenlof, K. H.: Nonstationary gravity wave forcing of the stratospheric zonal mean wind, J. Geophys. Res., 101, 23 465-23 476, 1996.

Alexander, M. J.: Interpretations of observed climatological patterns in stratospheric gravity wave variance, J. Geophys. Res., 103, 8627-8640, 1998.

Alexander, M. J., Tsuda, T., and Vincent, R. A.: Latitudinal variations observed in gravity waves with short vertical wavelengths, J. Atmos. Sci., 59, 1394-1404, 2002.

Allen, S. J. and Vincent R. A.: Gravity wave activity in the lower atmosphere: Seasonal and latitudinal variations, J. Geophys. Res., 100, 1327-1350, 1995.

Beres, J. H., Garcia, R. R., Boville, B. A., and Sassi, F.: Implementation of a gravity wave source spectrum parameterization dependent on the properties of convection in the Whole Atmosphere Community Climate Model (WACCM), J. Geophys. Res., 110, D10108, doi:10.1029/2004JD005504, 2005.

Chandra, S., Fleming, E. L., Schoeberl, M. R., and Barnett, J. J.: Monthly mean climatology of temperature, wind, geopotential height, and pressure for 0-120 km, Adv. Space Res., 10, 3-12, 1990.

Chun, H. Y. and Baik, J. J.: Momentum flux by thermally induced internal gravity waves and its approximation for large-scale models, J. Atmos. Sci., 55, 3299-3310, 1998.

Chun, H. Y. and Baik, J. J.: An updated parameterization of convectively forced gravity wave drag for use in large-scale models, J. Atmos. Sci., 59, 1006-1017, 2002.

Cot, C.: Equatorial mesoscale wind and temperature fluctuations in the lower atmosphere, J. Geophys. Res., 106, 1523-1532, 2001.
Coy, L. and Swinbank, R.: Characteristics of stratospheric winds and temperatures produced by data assimilation, J. Geophys. Res., 102, 25 763-25 781, 1997.

Dewan, E. M., Picard, R. H., O’Neil, R. R., Gardiner, H. A., Gibson, J., Mill, J. D., Richards, E., Kendra, M., and Gallery, W. O.: MSX satellite observations of thunderstorm-generated gravity waves in mid-wave infrared images of the upper stratosphere, Geophys. Res. Lett., 25, 939-942, 1998.

Eckermann, S. D. and Preusse, P.: Global measurements of stratospheric mountain waves from space, Science, 286, 1534-1537, 1999.

Eckermann, S. D., Broatman, D., Tan, K. A., Preusse, P., and Bacmeister, J. T.: Mountain Waves in the Stratosphere, NRLReview, 73-84, 2000.

Ern, M., Preusse, P., Alexander, M. J., and Warner, C. D.: Absolute values of gravity wave momentum flux derived from satellite data, J. Geophys. Res., 109, D20103, doi:10.1029/2004JD004752, 2004.

Ern, M., Preusse, P., and Warner, C. D.: A comparison between CRISTA satellite data and Warner and McIntyre gravity wave parameterization scheme: horizontal and vertical wavelength filtering of gravity wave momentum flux, Adv. Space Res., 35, 20172023, doi:10.1016/j.asr.2005.04.109, 2005.

Fels, S. B.: A parameterization of scale-dependent radiative damping rates in the middle atmosphere, J. Atmos. Sci., 39, 1141$1152,1982$.

Fels, S. B.: The radiative damping of short vertical scale waves in the mesosphere, J. Atmos. Sci., 41, 1755-1764, 1984.

Fritts, D. C. and VanZandt, T. E.: Spectral estimates of gravity wave energy and momentum fluxes. Part I: Energy dissipation, acceleration, and constraints, J. Atmos. Sci., 50(22), 3685-3694, 1993.

Fritts, D. C. and Alexander, M. J.: Gravity wave dynamics and effects in the middle atmosphere, Rev. Geophys., 41(1), 1003, doi:10.1029/2001RG000106, 2003.

Gardner, C. S.: Diffusive filtering theory of gravity wave spectra in the atmosphere, J. Geophys. Res., 99, 20 601-20 622, 1994.

Hertzog, A. and Vial, F.: A study of the dynamics of the equatorial lower stratosphere by use of ultra-long-duration balloons, 2, Gravity waves, J. Geophys. Res., 106, 22 745-22 761, 2001.

Hertzog, A., Souprayen, C., and Hauchecorne, A.: Measurements of gravity wave activity in the lower stratosphere by Doppler lidar, J. Geophys. Res., 106, 7879-7890, 2001.

Hertzog, A., Vial, F., Mechoso, C. R., Basdevant, C., and Cocquerez, P.: Quasi-Lagrangian measurements in the lower stratosphere reveal an energy peak associated with near-inertial waves, Geophys. Res. Lett., 29, 1229-1232, 2002.

Hines, C. O.: Doppler-spread parameterization of gravity-wave momentum deposition in the middle atmosphere. Part 1: Basic formulation, J. Atmos. Solar-Terr. Phys., 54, 371-386, 1997.

Hines, C. O.: Doppler-spread parameterization of gravity-wave momentum deposition in the middle atmosphere. Part 2: Broad and quasi monochromatic spectra and implementation, J. Atmos. Solar-Terr. Phys., 54, 387-400, 1997.

Hocking, W. K.: A new approach to momentum flux determination using SKiYMET meteor radars, Ann. Geophys., 23, 2433-2439, 2005 , http://www.ann-geophys.net/23/2433/2005/.

Jiang, J. H., Eckermann, S. D., Wu, D. L., and Ma, J.: A search for mountain waves in MLS stratospheric limb radi- 
ances from the winter Northern Hemisphere: Data analysis and global mountain wave modeling, J. Geophys. Res., 109, D03107, doi:10.1029/2003JD003974, 2004.

Jiang, J. H., Wang, B., Goya, K., Hocke, K., Eckermann, S. D., Ma, J., Wu, D. L., and Read, W. J.: Geographical distribution and interseasonal variability of tropical deep convection: UARS MLS observations and analyses, J. Geophys. Res., 109, D03111, doi:10.1029/2003JD003756, 2004.

Kim, Y. J., Eckermann, S. D., and Chun, H. Y.: An overview of the past, present and future of gravity-wave drag parameterization for numerical climate and weather prediction models, Atmos. Ocean, 41, 65-98, 2003.

Lindzen, R. S.: Turbulence and stress owing to gravity-wave and tidal breakdown, J. Geophys. Res., 86, 9707-9714, 1981.

Marks, C. J. and Eckermann, S. D.: A 3-dimensional nonhydrostatic ray-tracing model for gravity-waves - formulation and preliminary-results for the middle atmosphere, J. Atmos. Sci., 52, 1959-1984, 1995.

McFarlane, N. A.: The effect of orographically excited wave drag on the general circulation of the lower stratosphere and troposphere, J. Atmos. Sci., 44, 1775-1800, 1987.

Medvedev, A. S. and Klaassen, G. P.: Vertical evolution of gravity wave spectra and the parameterization of associated wave drag, J. Geophys. Res., 100, 25 841-25 853, 1995.

Nastrom, G. D., VanZandt, T. E., and Warnock, J. M.: Vertical wavenumber spectra of winds and temperature from highresolution balloon soundings over Illinois, J. Geophys. Res., 102, 6685-6701, 1997.

Oberheide, J., Lehmacher, G. A., Offermann, D., Grossmann, K. U., Manson, A. H., Meek, C. E., Schmidlin, F. J., Singer, W., Hoffmann, P., and Vincent, R. A.: Geostrophic wind fields in the stratosphere and mesosphere from satellite data, J. Geophys. Res., 107, 8175, doi:10.1029/2001JD000655, 2002.

Offermann, D., Grossmann, K. U., Barthol, P., Knieling, P., Riese, M., and Trant, R.: CRyogenic Infrared Spectrometers and Telescopes for the Atmosphere (CRISTA) experiment and middle atmosphere variability, J. Geophys. Res., 104, 16311-16325, 1999.

Preusse, P., Eidmann, G., Eckermann, S. D., Schaeler, B., Spang, R., and Offermann, D.: Indications of convectively generated gravity waves in CRISTA temperatures, Adv. Space Res., 27, 1653-1658, 2001.

Preusse, P., Dörnbrack, A., Eckermann, S. D., Riese, M., Schaeler, B., Bacmeister, J. T., Broutman, D., and Grossmann, K. U.: Space based measurements of stratospheric mountain waves by CRISTA, 1. Sensitivity, analysis method and a case study, J. Geophys. Res., 107, 8178, doi:10.1029/2001JD000699, 2002.

Preusse, P., Ern, M., Grossmann, K. U., and Mergenthaler, J. L.: Seasonal variations of gravity wave variance inferred from CLAES, Proc. SPIE, 5235, 288-297, 2004.
Preusse, P., Ern, M., Eckermann, S. D., Warner, C. D., Picard, R. H., Knieling, P., Krebsbach, M., Russell, J. M., Mlynczak, M. G., Mertens, C. J., and Riese, M.: Tropopause to mesopause gravity waves in August: measurement and modeling, J. Atmos. SolarTerr. Phys., in press, 2006.

Riese, M., Spang, R., Preusse, P., Ern, M., Jarisch, M., Offermann, D., and Grossmann, K. U.: Cryogenic Infrared Spectrometers and Telescopes for the Atmosphere (CRISTA) data processing and atmospheric temperature and trace gas retrieval, J. Geophys. Res., 104, 16349-16367, 1999.

Riese, M., Friedl-Vallon, F., Spang, R., Preusse, P., Schiller, C., Hoffmann, L., Konopka, P., Oelhaf, H., von Clarmann, T., and Höpfner, M.: GLObal limb Radiance Imager for the Atmosphere (GLORIA): Scientific objectives, Adv. Space Res., 36, 989-995, doi:10.1016/j.asr.2005.04.115, 2005.

Sato, K.: Small-scale wind disturbances observed by the MU radar during the passage of Typhoon Kelly, J. Atmos. Sci., 50, 518537, 1993.

Sato, K.: A statistical study of the structure, saturation and sources of inertio-gravity waves in the lower stratosphere observed with the MU radar, J. Atmos. Solar-Terr. Phys., 56, 755-774, 1994.

Swinbank, R. and O'Neill, A.: A stratosphere-troposphere data assimilation system, Mon. Weather Rev., 122, 686-702, 1994.

Tsuda, T., Inoue, T., Fritts, D. C., VanZandt, T. E., Kato, S., Sato, T., and Fukao, S.: MST radar observations of a saturated gravity wave spectrum, J. Atmos. Sci., 46, 2440-2447, 1989.

Tsuda, T., VanZandt, T. E., Mizumoto, M., Kato, S., and Fukao, S.: Spectral analysis of temperature and Brunt-Vaisala frequency fluctuations observed by radiosondes, J. Geophys. Res., 96, 17 265-17 278, 1991.

Tsuda, T. and Hocke, K.: Vertical wave number spectrum of temperature fluctuations in the stratosphere using GPS occultation data, J. Meteorol. Soc. Japan, 80, 925-938, 2002.

Tsuda T., Ratnam, M. V., May, P. T., Alexander, M. J., Vincent, R. A., and MacKinnon, A.: Characteristics of gravity waves with short vertical wavelengths observed with radiosonde and GPS occultation during DAWEX (Darwin Area Wave Experiment), J. Geophys. Res., 109, D20S03, doi:10.1029/2004JD004946, 2004.

VanZandt, T. E.: A universal spectrum of buoyancy waves in the atmosphere, Geophys. Res. Lett., 9, 575-578, 1982.

Warner, C. D. and McIntyre, M. E.: On the propagation and dissipation of gravity wave spectra through a realistic middle atmosphere, J. Atmos. Sci., 53, 3213-3235, 1996.

Warner, C. D. and McIntyre, M. E.: Toward an ultra-simple spectral gravity wave parameterization for general circulation models, Earth Planets Space, 51, 475-484, 1999.

Warner, C. D. and McIntyre, M. E.: An ultrasimple spectral parameterization for nonorographic gravity waves, J. Atmos. Sci., 58, 1837-1857, 2001. 